\title{
Gray matter volumetric abnormalities associated with the onset of psychosis
}

\author{
Wi Hoon Jung 1,2, Stefan Borgwardt ${ }^{3,4}$, Paolo Fusar-Poli ${ }^{4}$ and Jun Soo Kwon ${ }^{1,2,5,6 *}$ \\ ${ }^{1}$ Interdisciplinary Program in Neuroscience, Seoul National University, Seoul, South Korea \\ 2 Institute of Human Behavioral Medicine, Seoul National University-MRC, Seoul, South Korea \\ ${ }^{3}$ Department of Psychiatry, University of Basel, Basel, Switzerland \\ ${ }^{4}$ Department of Psychosis Studies, Institute of Psychiatry, King's College, London, UK \\ ${ }^{5}$ Department of Psychiatry and Behavioral Sciences, Seoul National University College of Medicine, Seoul, South Korea \\ ${ }^{6}$ Brain and Cognitive Sciences-WCU program, College of Natural Sciences, Seoul National University, Seoul, South Korea
}

\section{Edited by:}

Bernat Kocsis, Harvard Medical School, USA

Reviewed by:

Bernat Kocsis, Harvard Medical School, USA

Steven R. Laviolette, University of

Western Ontario, Canada

\section{*Correspondence:}

Jun Soo Kwon, Department of

Psychiatry and Behavioral Sciences,

Seoul National University College of

Medicine, 101 Daehak-no,

Chongno-gu, Seoul 110-744, South

Korea.

e-mail: kwonjs@snu.ac.kr
Patients with psychosis display structural brain abnormalities in multiple brain regions. The disorder is characterized by a putative prodromal period called ultra-high-risk (UHR) status, which precedes the onset of full-blown psychotic symptoms. Recent studies on psychosis have focused on this period. Neuroimaging studies of UHR individuals for psychosis have revealed that the structural brain changes observed during the established phases of the disorder are already evident prior to the onset of the illness. Moreover, certain brain regions show extremely dynamic changes during the transition to psychosis. These neurobiological features may be used as prognostic and predictive biomarkers for psychosis. With advances in neuroimaging techniques, neuroimaging studies focusing on gray matter abnormalities provide new insights into the pathophysiology of psychosis, as well as new treatment strategies. Some of these novel approaches involve antioxidants administration, because it is suggested that this treatment may delay the progression of UHR to a full-blown psychosis and prevent progressive structural changes. The present review includes an update on the most recent developments in early intervention strategies for psychosis and potential therapeutic treatments for schizophrenia. First, we provide the basic knowledge of the brain regions associated with structural abnormalities in individuals at UHR. Next, we discuss the feasibility on the use of magnetic resonance imaging (MRI)-biomarkers in clinical practice. Then, we describe potential etiopathological mechanisms underlying structural brain abnormalities in prodromal psychosis. Finally, we discuss the potentials and limitations related to neuroimaging studies in individuals at UHR.

Keywords: psychosis, biomarker, gray matter, magnetic resonance imaging, schizophrenia, high-risk subjects

\section{INTRODUCTION}

Schizophrenia is the most disabling psychotic illness that affects approximately $1 \%$ of individuals worldwide. The disorder is characterized by a prodromal period that precedes the onset of full-blown psychotic symptoms, showing abnormalities in socialization and thinking (Yung and McGorry, 1996). Recent studies on psychosis have focused on the early course of the disorder (McGorry et al., 2008). The ultimate goal of researchers is to determine vulnerability- and disease-specific biological markers in an effort to predict the onset or course of the illness.

Over the past three decades, multiple neuroimaging studies have revealed brain abnormalities in patients with psychotic disorders. The notion that brain abnormalities contribute to the etiology of psychosis was initially proposed by Kraepelin (1919/1971) and Bleuler (1950) more than 110 years ago. This concept, however, was largely discredited during the twentieth century. A renewed interest in psychosis was generated after the first CT study by Johnstone et al. (1976). The first magnetic resonance imaging (MRI) study in patients with schizophrenia was published by
Smith et al. (1984). Since then, the investigation of brain structure abnormalities associated with psychosis has flourished. MRI is the most established and used technique of for the investigation of the human brain structure in vivo. Over the past 27 years, MRI studies in patients with psychosis have played a pivotal role in providing support for the argument that brain abnormalities are associated with the disease. Moreover, this period has offered tremendous knowledge about the neurobiological basis of psychosis. Patients with psychosis have been shown to have enlarged lateral ventricles and reduced volume in the hippocampus, superior temporal gyrus (STG), and prefrontal cortices (PFC; Shenton et al., 2001; MacDonald and Schulz, 2009). Some discussion however was raised regarding the potential confounding role played by illness duration and antipsychotic treatment. Interestingly, some of these structural abnormalities are also observed in first-episode patients (FEP; Steen et al., 2006) who have a short duration of illness and a minimal exposure to antipsychotic treatment. Recently, new approaches that study individuals at high-risk for the disease have been used to better circumvent these problems and address this issue. 
Specifically, two approaches focusing on high-risk subjects have been used to elucidate vulnerability to psychosis and minimizing confounding variables including neurodegenerative progress of the disease, institutionalization, and long-term treatment with antipsychotic medication. The genetic-high-risk (GHR) approach focuses on non-psychotic first-degree relatives of patients with schizophrenia, exploring trait markers of genetic vulnerability for the disorder, called endophenotypes. Alternatively, the clinical high-risk [CHR; also called ultra-high-risk (UHR), or at risk mental state (ARMS)] strategy focuses on individuals presenting with subthresholded psychotic features: (i) attenuated psychotic symptoms (APS), (ii) brief limited intermitted psychotic symptoms (BLIPS), and (iii) trait-plus-state risk factors [i.e., recent decline in social functioning and genetic risk for psychosis, genetic risk and deterioration syndrome (GRD; Yung et al., 2003]. Historically, the development of UHR criteria was introduced by Yung and McGorry, 1996; for a comprehensive state-of-the-art review on the high-risk state see, Fusar-Poli et al., 2012a). Research in the UHR field has progressed to the point that a new UHR diagnostic category is being considered for introduction in the forthcoming DSM-5 (Fusar-Poli and Yung, 2012). A recent meta-analysis in over than $2500 \mathrm{CHR}$ subjects has showed that there is a consistent transition risk across different diagnostic criteria ranging from $18 \%$ at 6 months up to $36 \%$ after 3 years (Fusar-Poli et al., 2012b). The majority (73\%) of these high-risk subject who will later transit to psychosis will develop a schizophrenic psychosis as compared to affective psychoses (11\%, risk ratio 5.44; Fusar-Poli et al., 2012c). The high-risk state for psychosis is also associated with impaired quality of life and subtle impairments in cognitive functioning (Fusar-Poli et al., 2012d). In this scenario, neurobiological markers of liability to psychosis and transition to full illness can augment the predictive validity of current psychopathologically based criteria (Fusar-Poli and Broome, 2006; McGuire et al., 2008). To address this issue, neuroimaging studies of UHR patients may describe neurobiological markers of illness state as well as trait markers. However, despite the large number of structural imaging investigations in established psychosis and in UHR subjects, clinical applications for psychiatric neuroimaging are still lacking (Kloppel et al., 2012).

In the present review, recent MRI findings of UHR patients are critically reviewed along with multiple structural brain measures (regional brain volume and cortical thickness) suggesting that gray matter volume (GMV) abnormalities are associated with the onset of psychosis. Therefore, this review focuses on studies of MRI changes in UHR patients. The MRI studies discussed in this review are selected on a critical review of the available literature and they are listed in Table 1. The present article is not intended as a thorough review of all structural MRI studies in UHR patients [Wood et al., 2008; Pantelis et al., 2009; Jung et al., 2010; Smieskova et al., 2010; Borgwardt et al., 2011; Fusar-Poli et al., 2011a; Gogtay et al., 2011; for meta-analyses of structural findings in subjects at high clinical risk for psychosis (see FusarPoli et al., 2011b, 2012f) in subjects at genetic risk for psychosis (see Fusar-Poli et al., 2012e) for functional meta-analyses in subjects at clinical risk for psychosis (see Fusar-Poli et al., 2007; Fusar-Poli and Meyer-Lindenberg, 2012a,b)]. Rather, this review (i) provides basic knowledge of the brain regions associated with structural abnormalities in UHR patients; (ii) focuses on the use of MRI-biomarkers in clinical practice; (iii) suggests potential etiopathological mechanisms underlying structural brain abnormalities in prodromal psychosis; and (iv) discusses the potentials and limitations related to neuroimaging studies in individuals at UHR.

\section{GM ABNORMALITIES IN INDIVIDUALS AT UHR CROSS-SECTIONAL COMPARISON BETWEEN INDIVIDUALS AT UHR AND HEALTHY CONTROLS}

Magnetic resonance imaging studies of patients with established psychosis have revealed brain structural abnormalities associated with the disorder. Individuals at UHR, however, may demonstrate some specific markers of potential vulnerability to psychosis. Accumulating data has revealed that neurobiological abnormalities in UHR subjects are qualitatively similar to but less severe than are those in patients with established psychosis.

Previous studies employing region of interest (ROI) or wholebrain voxel-based morphometric (VBM) methods have reported reduced hippocampal GMV in UHR individuals compared with healthy controls (Phillips et al., 2002; Wood et al., 2005, 2010; Borgwardt et al., 2007a; Hurlemann et al., 2008; Buehlmann et al., 2010; Witthaus et al., 2010), although results have been inconsistent (Velakoulis et al., 2006). Studies have shown that UHR individuals displayed deficits in hippocampal-associated verbal memory (Hurlemann et al., 2008). Wood et al. (2005) reported decreased hippocampal volume in UHR patients without a family history of psychosis compared with individuals with a family history of psychosis, suggesting that non-specific environmental factors are more highly associated with morphological abnormalities than are genetic factors.

Prefrontal cortex abnormalities in UHR patients have been reported in the lateral PFC (Meisenzahl et al., 2008; Koutsouleris et al., 2009a; Witthaus et al., 2009), medial PFC, and ACC (Borgwardt et al., 2007a; Witthaus et al., 2009; Jung et al., 2011; Choi et al., 2012). Moreover, using VBM and functional MRI (fMRI) techniques, reduced GMV in the middle frontal gyrus was correlated with reduced neural activity in this region (Fusar-Poli et al., 2011c). Deficits in the ACC may provide evidence of early neurodevelopmental anomalies in schizophrenia, as reports have indicated alterations in the pattern of cortical folding in this region (Yücel et al., 2003). Altered cortical folding patterns in UHR individuals without a family history of psychosis, compared with individuals with a family history, suggested involvement of environmental rather than genetic factors (Wood et al., 2005). Specifically, abnormalities in the midline cortical structures (i.e., medial PFC and ACC) in UHR patients may contribute to their disrupted sense of self (Nelson et al., 2009). Recently, UHR individuals were shown to have aberrant connectivity in the default mode network, which is involved in self-referential processing (Shim et al., 2010). Reduced GMV in the parietal area in UHR patients was also observed (Fusar-Poli et al., 2011d), and cortical thinning in the parietal regions in UHR patients was reported (Jung et al., 2011). However, Haller et al. (2009) reported no differences in cortical thickness, but did find differences in cortical asymmetry in the parietal, insular, and occipitotemporal gyri between UHR individuals and controls. A recent magnetoencephalography (MEG) 


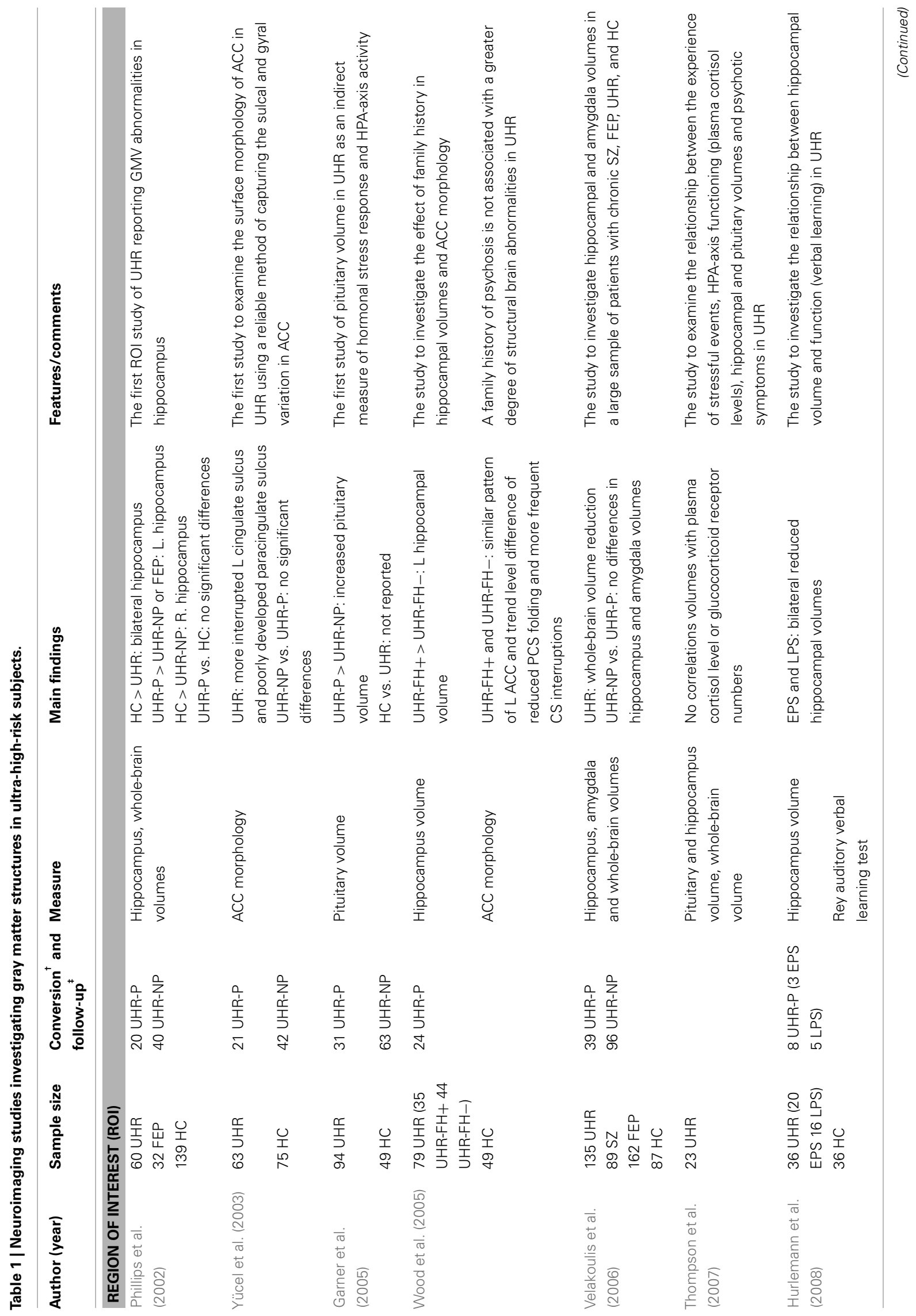



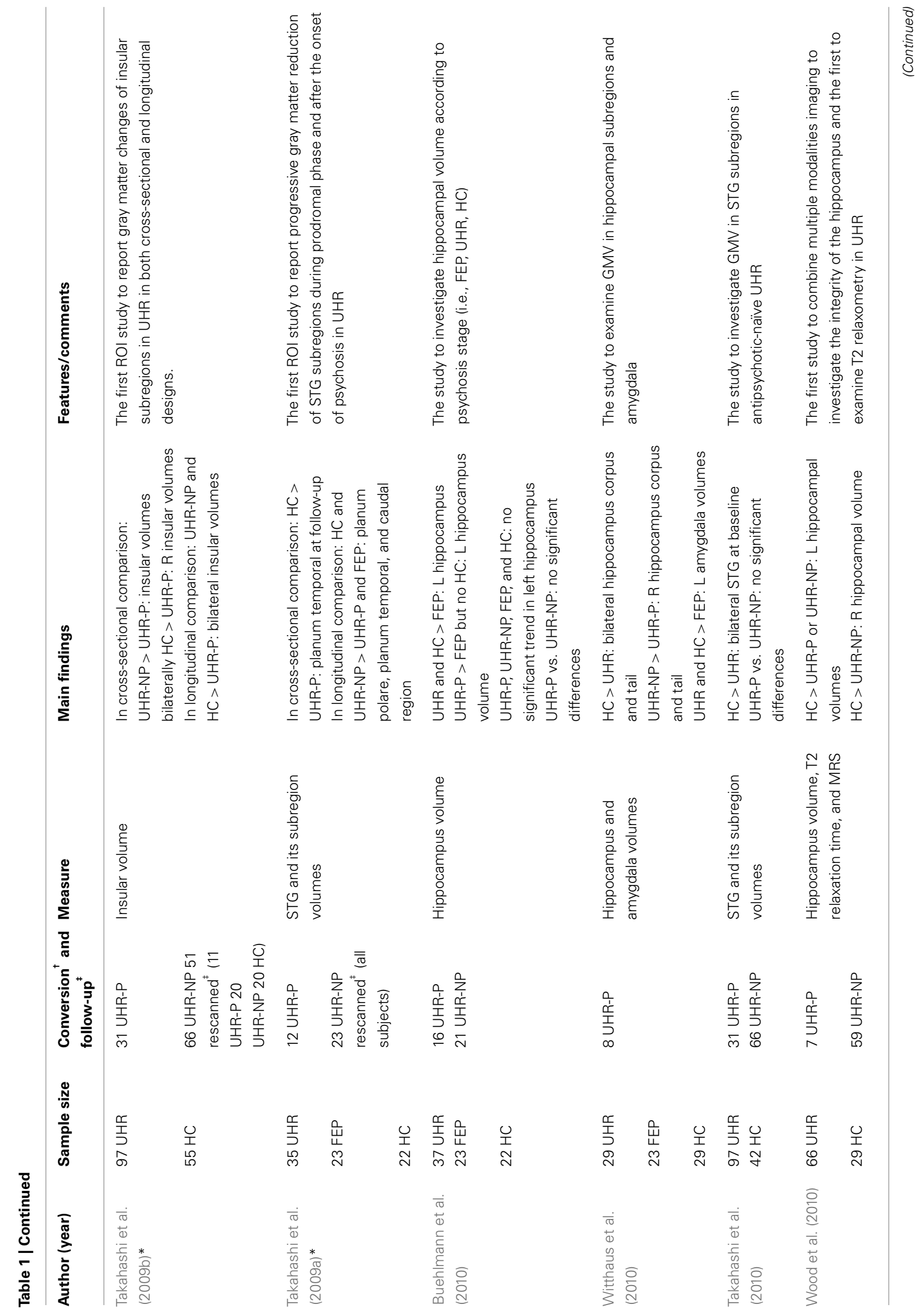

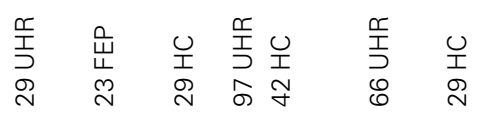




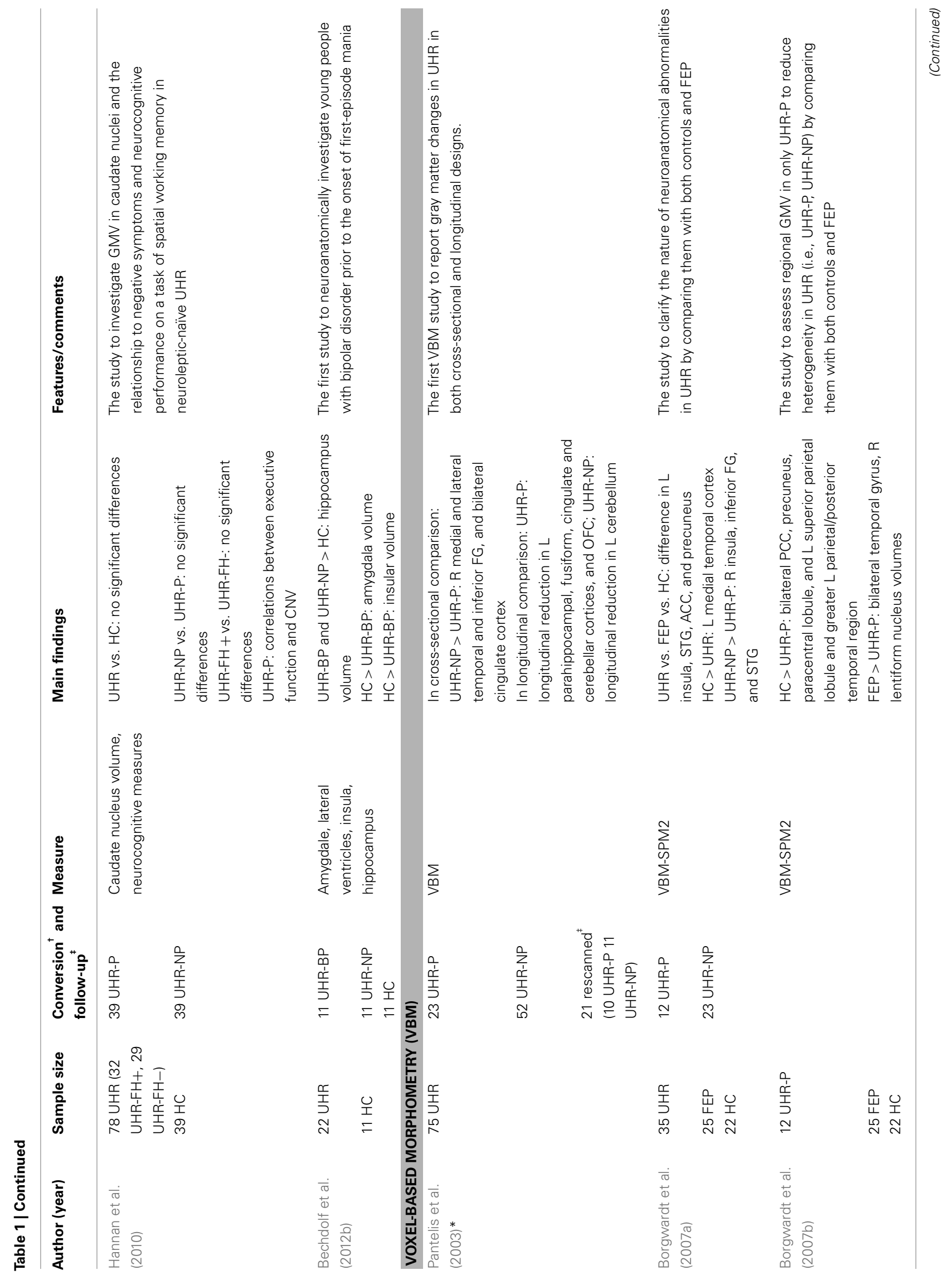




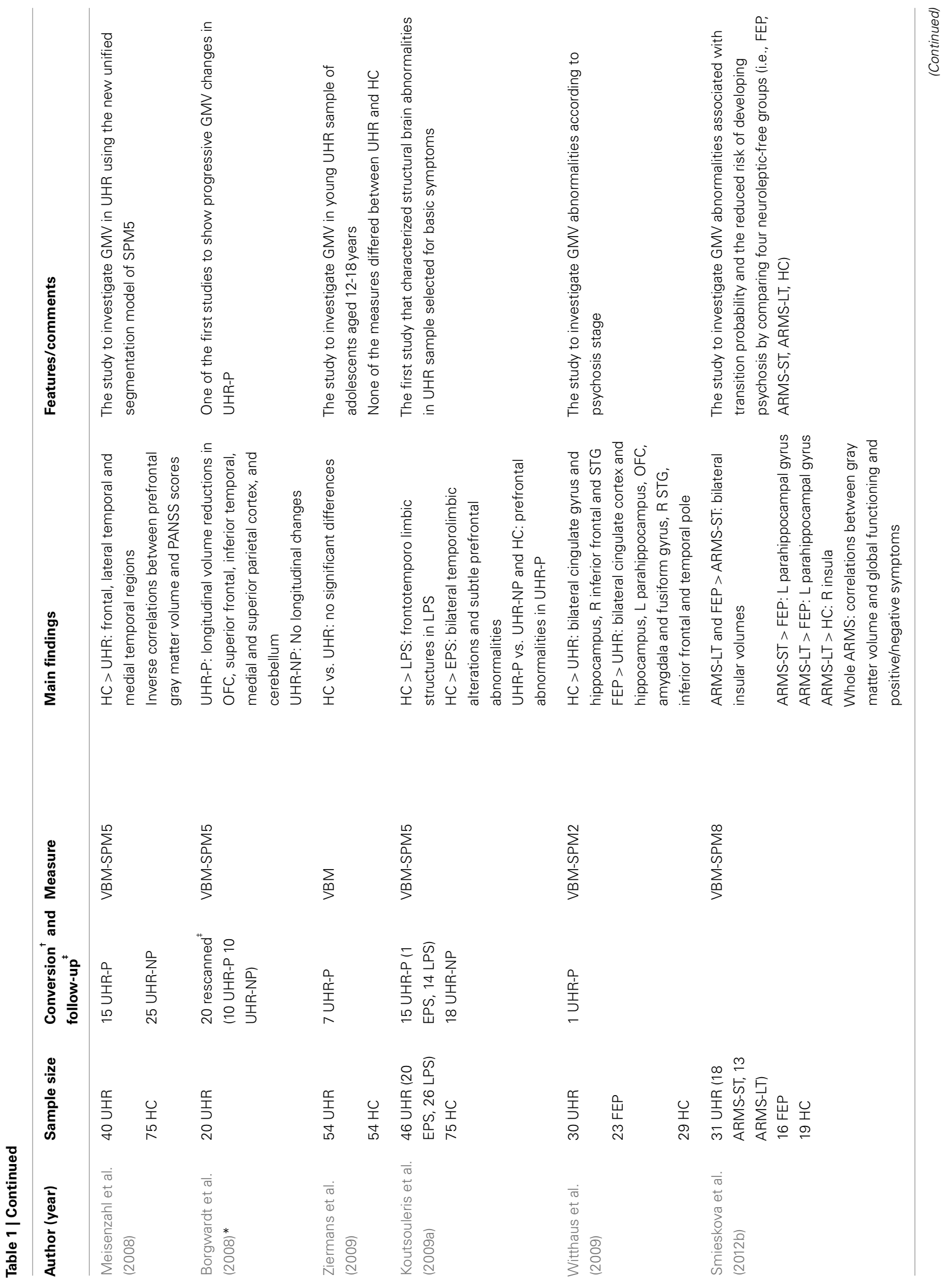




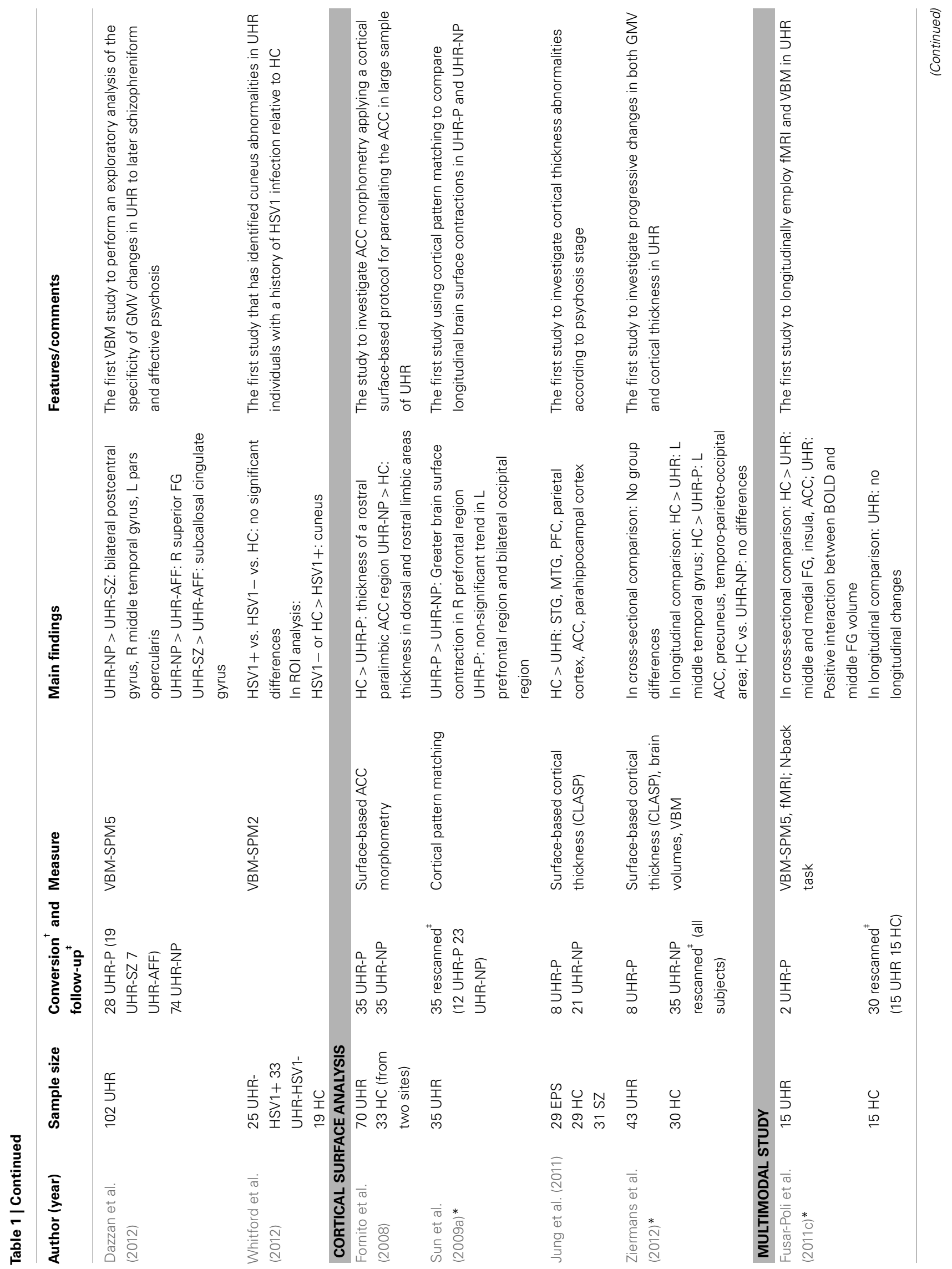




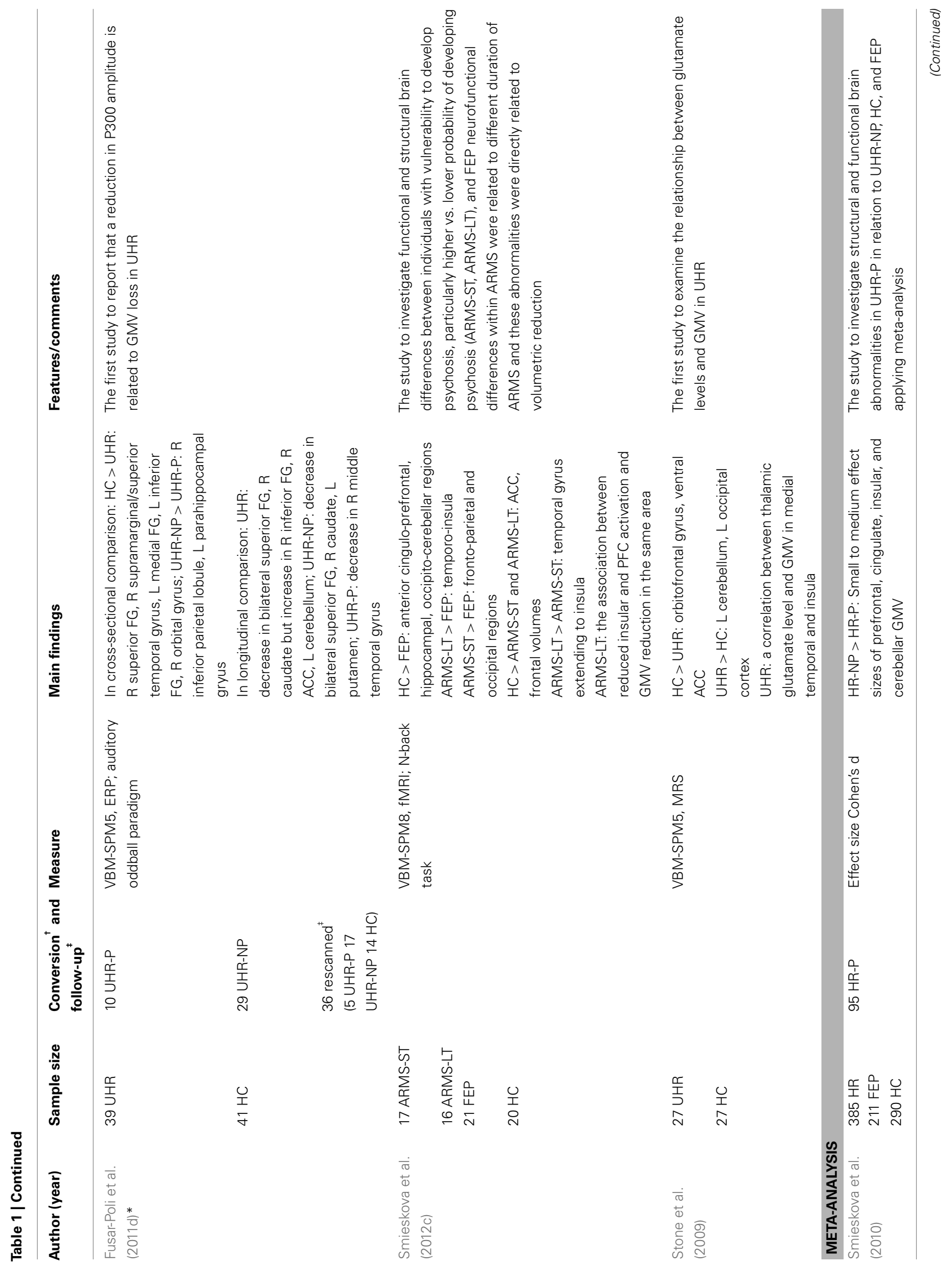




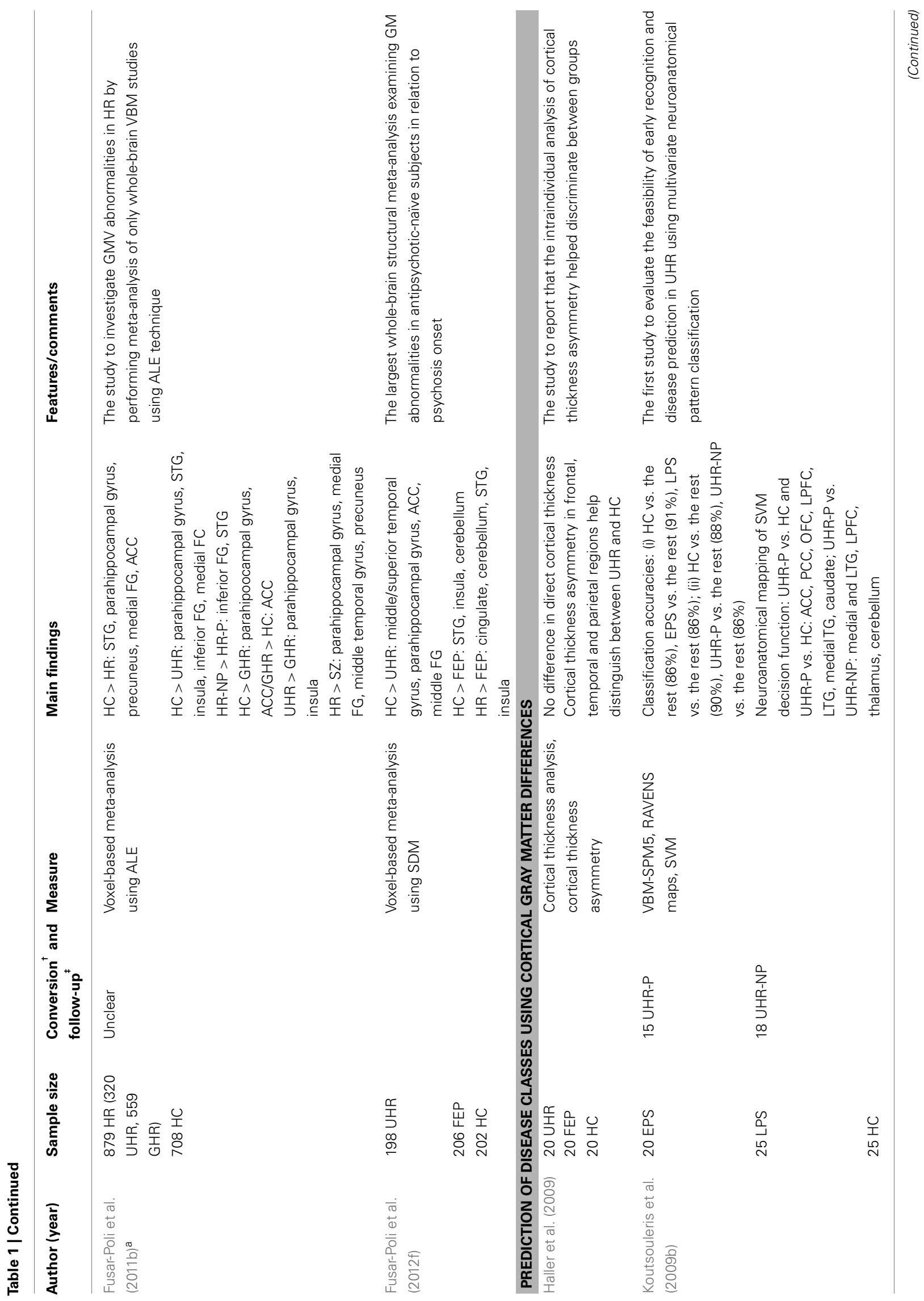




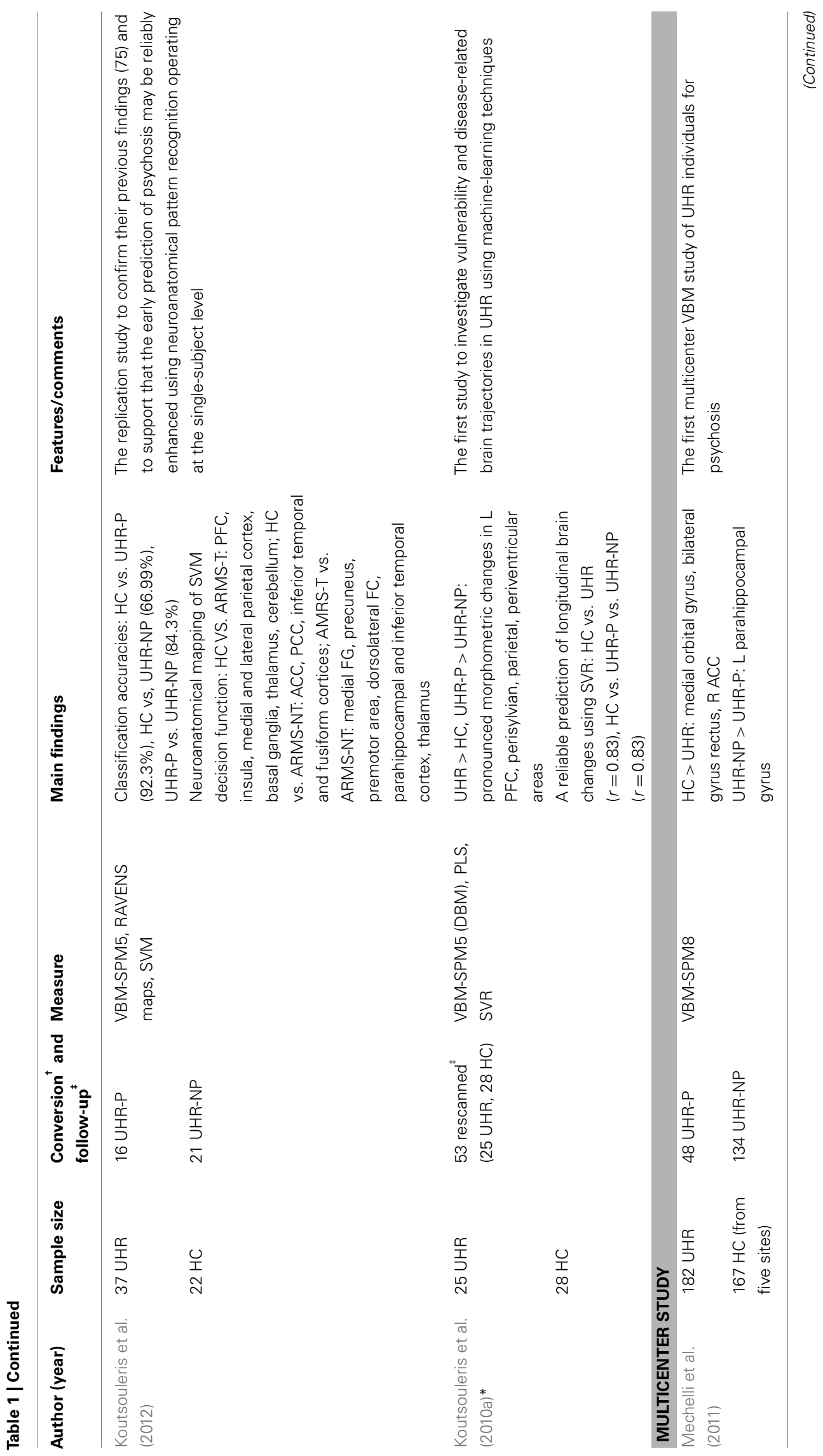




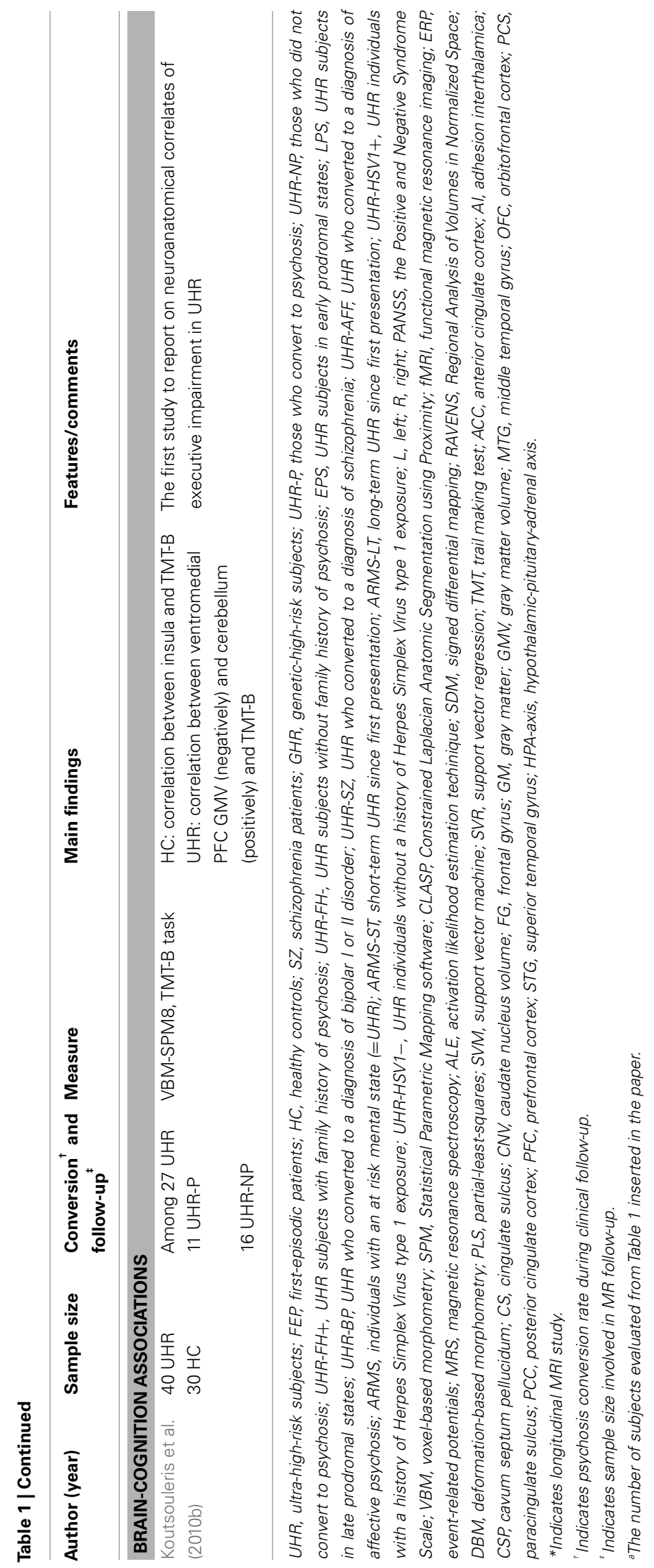


study in UHR patients showed aberrant alpha modulation related to selective attention, particularly in the parieto-occipital region (Koh et al., 2011). A multimodal EEG-MRI study reported a relationship between P300 amplitude and GMV in the supramarginal gyrus in UHR patients (Fusar-Poli et al., 2011d).

Volumetric abnormalities in the lateral temporal lobe, particularly the STG, in UHR patients have frequently been reported. The STG processes sounds and social information and is related to functional deficits, including auditory hallucinations and thought disorders (Allen, 2008; Sun et al., 2009b). Several ROI and VBM studies in UHR patients have reported reduced STG volume (Borgwardt et al., 2007a; Koutsouleris et al., 2009a; Witthaus et al., 2009; Takahashi et al., 2010), although these findings are inconsistent (Takahashi et al., 2009a). Recent studies of surface-based cortical thickness were consistent with previous findings and indicated reduced STG volume in UHR patients (Jung et al., 2011). MEG studies have identified aberrant auditory processing in UHR individuals, including defects in N1m adaptation, the magnetic counterpart of MMN (MMNm), and correlations between MMNm and clinical symptoms (Shin et al., 2009, 2012).

Aberrant GMV in limbic system regions such as the insula and amygdala has also been reported. UHR individuals showed reduced insular volume (Borgwardt et al., 2007a,b; Takahashi et al., 2009b) but no differences in amygdala volume (Velakoulis et al., 2006; Witthaus et al., 2009). A recent study investigated the volume of the caudate nuclei using ROI techniques (Hannan et al., 2010). Caudate nuclei volumes did not differ significantly between UHR individuals and healthy controls.

Given that the medial PFC, ACC, inferior frontal gyrus, insula, and STG are involved in social cognitive processes (Blakemore, 2008), UHR individuals may display abnormal social functioning. There is significant evidence to support this hypothesis [i.e., impaired social functioning (Shim et al., 2008) and social cognition (Chung et al., 2008)]. Patients with psychosis have alterations in facial processing (Shin et al., 2008). Interestingly, UHR individuals also display similar deficits (Kim et al., 2010), suggesting a role for social dysfunction in psychosis.

\section{CROSS-SECTIONAL COMPARISON BETWEEN INDIVIDUALS AT UHR-P AND UHR-NP}

Individuals at UHR are a heterogeneous group that includes individuals who later develop psychosis (UHR-P) and those who do not (UHR-NP). Some brain abnormalities may reflect an underlying vulnerability for psychosis, whereas others may be associated with progression due to an acute illness. Therefore, differences in GMV between individuals at UHR-P and UHR-NP are of specific interest.

In ROI studies, individuals at UHR-P displayed smaller insular (Takahashi et al., 2009b) and hippocampal GMV (Wood et al., 2010) and increased pituitary volume (Garner et al., 2005). Phillips et al. (2002) reported greater hippocampal volume in UHR-P patients compared with UHR-NP patients and FEP. No GMV differences were noted between UHR-P and UHR-NP in several brain regions including the STG (Takahashi et al., 2010), hippocampus (Buehlmann et al., 2010), amygdala (Velakoulis et al., 2006), caudate nucleus (Hannan et al., 2010), or in ACC cortical folding patterns (Yücel et al., 2003). However, the caudate nucleus volume in UHR-P patients was positively correlated with total errors in spatial working memory and verbal fluency performance (Hannan et al., 2010). In VBM studies, UHR-P patients displayed smaller GMV in the insula and in the ACC compared with UHR-NP patients (Pantelis et al., 2003; Borgwardt et al., 2007a). Borgwardt et al. (2007b) found no differences in these two regions between individuals at UHR-P and FEP. Reductions in the PFC and orbitofrontal cortex, however, were noted (Koutsouleris et al., 2009a; Dazzan et al., 2012). Fornito et al. (2008) assessed cortical thickness in the ACC in UHR patients and found that UHR-P individuals showed cortical thinning in the rostral paralimbic region. A recent study reported decreased cortical thickness in the ACC, PFC, STG, and inferior temporal cortex in UHR-P individuals (Jung et al., 2011). Moreover, UHR-P individuals displayed poor social cognition (Kim et al., 2011) and social functioning compared with UHR-NP individuals (Jang et al., 2011).

\section{LONGITUDINAL COMPARISON}

Longitudinal studies have focused on the pathophysiological nature of brain abnormalities around the time of the onset of illness. Using the VBM approach, Pantelis et al. (2003) reported that UHR-P patients displayed longitudinal reductions in the orbitofrontal, cerebellar, fusiform, and parahippocampal cortices and the cingulate gyrus. Borgwardt et al. (2008) reported progressive volume reductions in the orbitofrontal cortex and cerebellum, as well as the superior frontal, inferior temporal, and superior parietal cortices and the precuneus. ROI studies have found greater reductions in the insula (Takahashi et al., 2009b) and STG (Takahashi et al., 2009a) in UHR-P patients compared with patients at UHR-NP. Using cortical pattern matching techniques, Sun et al. (2009a) revealed greater brain contraction in the PFC in UHR$\mathrm{P}$ patients compared with that in UHR-NP patients. A recent surface-based cortical thickness study found that UHR-P individuals displayed cortical thinning in widespread regions in the ACC, precuneus, and temporo-parieto-occipital areas (Ziermans et al., 2012). Recent longitudinal multimodal studies have used MRI with other neuroimaging modalities. A longitudinal electroencephalogram (EEG)-VBM study reported progressive GM alterations in the PFC and subcortical areas, including the caudate, in patients at UHR, but no significant changes over time in the P300 amplitude during a task (Fusar-Poli et al., 2011d). UHR-P patients showed reduced GMV in the middle temporal gyrus, and UHR-NP patients showed decreased GMV in the superior frontal gyrus, caudate, and putamen between a baseline and follow-up session. In contrast, no significant differences in GMV were noted between the baseline and the follow-up scan in UHR patients in another longitudinal fMRI-VBM study from the same center (Fusar-Poli et al., 2011c). However, longitudinal increases in activation in the parahippocampus and ACC were noted. Taken together, these findings suggest that GMV abnormalities in some brain regions dynamically emerge during the acute process of transition to psychosis, particularly showing progressive changes in the frontal and temporal regions in UHR-P patients.

\section{VOXEL-WISE META-ANALYSES AND MULTICENTER STUDIES}

There is accumulating evidence for structural changes in UHR individuals. Moreover, researchers have recently conducted 
voxel-wise meta-analysis studies in UHR patients (Smieskova et al., 2010; Fusar-Poli et al., 2011b, 2012f). Smieskova et al. (2010) reported decreased PFC, cingulate, insula, and cerebellar GMV in UHR-P patients compared with UHR-NP patients. Methodological differences and different high-risk statuses (i.e., GHR, UHR) were noted between the studies. However, changes in the PFC, ACC, medial temporal, and cerebellar cortex may predict the development of psychosis in UHR patients. To address the heterogeneity across studies that use different methods, Fusar-Poli et al. (2011b) conducted a voxel-wise meta-analysis of wholebrain VBM studies in high-risk individuals including UHR or GHR. UHR patients compared with healthy controls displayed reduced GMV in the STG, parahippocampal region, precuneus, medial and middle frontal gyri, and the ACC. Compared with UHR-NP patients, UHR-P patients showed baseline GM reductions in the inferior frontal gyrus and the STG. UHR individuals relative to GHR displayed greater volume in the parahippocampal gyrus, insula, and STG and less volume in the ACC. Additionally, the study confirmed significant effects of atypical antipsychotic treatment on the inferior frontal gyrus and insula. To remove confounding effects of antipsychotic drugs, Fusar-Poli et al. (2012f) conducted an additional voxel-wise meta-analysis on VBM studies enrolling antipsychotic-naïve subjects. GMV reductions were found in the middle and superior temporal gyri, hippocampal regions, and middle frontal gyrus in patients at UHR, and in the STG, insula, and cerebellum in FEP patients. Compared with UHR subjects, FEP subjects showed GM decreases in the STG, ACC, insula, and cerebellum. Moreover, an inverse correlation was noted between STG GMV and symptom severity, suggesting that the STG is associated with the onset of psychotic symptoms.

A multicenter study conducted on UHR patients collected data from five sites (subjects were 167 healthy controls, 48 UHR-P, 134 UHR-NP) and was recently published (Mechelli et al., 2011). The UHR group had less GMV in the frontal regions compared with controls. Compared with patients with UHR-NP, UHR-P patients had less GMV in the parahippocampal cortex.

\section{THE FEASIBILITY OF NEUROANATOMICAL BIOMARKERS}

Despite the convincing evidence from neuroimaging studies, the feasibility of using MRI-based data on structural changes remains far in the future for clinical practice in psychiatry. This is largely due to the overlap with anatomical variation within the normal range. Recently, advancements in computer visualization and neuroimaging techniques have raised intense interest in diagnostic MRI-based biomarkers. Particularly, multivariate classification approaches for pattern recognition of whole-brain data (i.e., support vector machine, SVM) are becoming increasingly popular in situations in which changes do not affect a single location but are expressed in a distinct pattern across the brain. Using advanced pattern recognition methods, Koutsouleris et al. (2009b) attempted to develop potential MRI-based biomarkers for the prediction of illness outcomes that are reliable and feasible in clinical practice. They convincingly demonstrated extensive GM distribution differences in various categories in individuals at risk for psychosis and morphological pattern differences between individuals at UHR-P and those at UHR-NP. The findings from this study have been replicated in independent populations (Koutsouleris et al., 2012). The expression of progressive dynamic changes in GMV during illness transition has been predicted using neuroanatomical pattern regression (i.e., support vector regression, SVR; Koutsouleris et al., 2010a).

Although the aforementioned studies showed the potential for early recognition and disease prediction in patients at UHR, it is unclear whether MRI-based biomarkers could generalize to clinical practice. There are some unresolved questions regarding the generalization of biomarkers (Kloppel et al., 2012). Given the high classification accuracy from the multivariate pattern recognition approach and the view that schizophrenia is a disorder of large-scale neurocognitive networks rather than specific regions (Menon, 2011), we suggest that neuroanatomical markers for psychosis may be associated with distinct coordinated patterns of cortical morphology rather than with abnormalities in specific regions per se. In this sense, the concepts of graph theory (He et al., 2008) and hyper-networks (Ha et al., 2009) may be applied to quantitatively characterize these complex anatomical patterns. Future studies should address issues of clinical and genetic heterogeneity between patients, including differences in symptoms, duration, medication, and genetic vulnerability, which may influence neurobiological substrates in a complex way. The human brain is not static, but dynamically changes throughout life. MRI-biomarkers for psychosis are also not static and may therefore indicate the magnitude of change over time.

\section{POTENTIAL ETIOPATHOLOGICAL MECHANISMS FOR GM ABNORMALITIES AND THERAPEUTIC IMPLICATIONS}

The underlying basis of GM abnormalities is currently unknown; however, alterations in brain processes involved in the pathology of psychosis may contribute to the structural abnormalities. Structural brain alterations in the fronto-temporal regions observed in patients with psychosis occur before the onset of full-blown psychosis. These regions are the same regions where lesions in animals result in striatal dopaminergic abnormalities (Flores et al., 1996; Lodge and Grace, 2007; Howes and Kapur, 2009). Striatal hyperdopaminergia has been postulated to be fundamental to the emergence of the psychotic symptoms (Kapur et al., 2005). Recent studies indicate that elevated striatal dopamine function predates the onset of psychosis in UHR subjects (Howes et al., 2009; Fusar-Poli et al., 2010a). It is thus conceived that striatal dopaminergic elevation is present in a compromised brain in psychosis and GM alterations may be associated with dopaminergic subcortical alterations (Howes and Kapur, 2009).

Based on findings from post-mortem studies, GMV alterations may be due to changes in neuronal, synaptic, and dendritic density, as well as increased afferentation in certain regions (Fornito et al., 2009). The combined volume of synapses, axons, and dendrites make up approximately $70 \%$ of the total GMV (Chklovskii et al., 2002; Fingerman et al., 2010). Abnormalities in specific region may result from different causes in different individuals (Fornito et al., 2009). Therefore, differences in developmental trajectories may result in significant differences between patients and controls (Fingerman et al., 2010). Recent works by Fornito et al. (2009) have helped to bridge the gap between neuroimaging and the neuropathology of schizophrenia, particularly in the ACC. Differences 
in cortical GMV may be due to differences in the neuropil (Sowell et al., 2003), where the synaptic connections between branches of axons and dendrites are formed. Therefore, excessive synaptic pruning during adolescence may contribute to the structural changes. However, the effects of synapse loss are less than the differences in GMV observed between patients and controls, as synaptic boutons constitute approximately $10 \%$ of GMV (Chklovskii et al., 2002; Fingerman et al., 2010). One-third of the signal in T1-imaged cortex is from white matter (Paus, 2005); therefore, myelination of excess connections (increased afferentation) during adolescence and early adulthood may contribute to the reductions in GMV observed on MRI (Benes et al., 1987). Any alterations in apoptotic mechanisms associated with cell death may also contribute to differences in GMV.

Therefore, it may be valuable to discuss the causes of aberrant apoptotic mechanisms in relation to contemporary theories of psychosis. Note that the following suggestions are purely speculative. One possibility is the involvement of immune and inflammatory mechanisms, which directly affect neuronal proliferation, differentiation, migration, and apoptosis (Ader, 2007). It has been hypothesized that viruses could directly damage brain structures, or a virus may come in contact with the fetal brain and induce a dysfunctional reaction in the immature immune system, leading to autoimmune pathology (Kirch, 1993). Among antibodies against neurotropic viruses, studies have focused on the family of herpes viruses. Exposures to herpes simplex virus type 1 (HSV1) in patients with UHR was associated with structural abnormalities in the cuneus, which is consistent with the region found in HSV1-infected patients with established schizophrenia (Whitford et al., 2012).

Another possibility is the experience of psychological stress around the time of illness onset (Lodge and Grace, 2011). Generally, one biological system proposed as the link between the psychological experience of stress and the development of psychosis is the hypothalamic-pituitary-adrenal (HPA) axis (Phillips et al., 2006). Garner et al. (2005) examined pituitary volume as an indirect measure of HPA-axis dysfunction and found a larger pituitary volume in UHR-P compared with UHR-NP patients. Thompson et al. (2007) found that compared with UHR-NP, UHR-P patients displayed lower plasma cortisol levels at baseline, although there was no correlation between these levels and stressful events, suggesting that HPA-axis dysfunction is involved in the development of psychosis.

Recent studies have suggested the role of stress in GMV alterations in corticostriatal-limbic regions associated with emotional regulation and cognitive control. This is consistent with studies in individuals at UHR, suggesting that these changes may serve to mediate vulnerability to later psychopathology. A significant relationship between cumulative adversity/stress and smaller GMV in the medial PFC, ACC, and insula was found in adults (Ansell et al., 2012). Exposure to childhood maltreatment was associated with reduction in corticostriatal-limbic GM (Edmiston et al., 2011). Moreover, the results of preliminary analyses showed different regional patterns of decreases in GM between males and females; females showed GM reductions in regions associated with emotional regulation, whereas males showed GM reductions in regions involved in impulse control. The authors speculated that vulnerabilities to mental illness in adolescence may be moderated by gender. These sexually dimorphic developmental trajectories are associated with sex hormones and may contribute to sex differences in psychiatric disorders. Pubertal hormones play a role in brain development during the transition from childhood to adolescence, including structural brain-circuit remodeling, myelination, neural pruning, apoptosis, and dendritic spine remodeling (Vigil et al., 2011). Indeed, estrogen has neuroprotective effects on the brain (Norbury et al., 2003). For example, it acts against several toxins that boost the production of free radicals (Simpkins et al., 1994) and may itself act as an antioxidant to protect membranes against reactive oxygen species (ROS) as byproducts of oxygen metabolism (Behl et al., 1995). There is a high consumption of oxygen and enrichment of polyunsaturated fatty acids (PUFAs) in the neuronal membrane. Therefore, the brain is highly susceptible to oxidative damage from free radical attack, called lipid peroxidation. Oxidative stress due to an excess of ROS or a decrease in antioxidant levels results in brain damage and can lead to neurodegenerative diseases. Estrogen may play a role as an antioxidant. Estrogen also modulates the neurochemical transmitter systems, including the serotonergic, cholinergic, noradrenergic, and dopaminergic systems (Norbury et al., 2003). These neurotransmitter systems are associated with the symptoms of schizophrenia. The effects of estrogen on neurotransmitters may partially explain why late-onset schizophrenia is more common in post-menopausal women (i.e., decline in levels of estrogen) and the disorder presents earlier in men than in women. This concept is known as the estrogen-protection hypothesis of schizophrenia (Riecher-Rossler and Kulkarni, 2011). Kulkarni et al. (2012) recently reviewed this hypothesis and suggested that estrogen may offer a novel therapeutic strategy. Alternatively, the testosteroneprotection hypothesis states that testosterone protects against schizophrenia by modulating the neurotransmitters implicated in the disorder (Duggal et al., 2002). Testosterone also plays a neuroprotective role in the brain (Hammond et al., 2001), and lower testosterone in patients with schizophrenia was associated with a greater number of negative symptoms (Akhondzadeh et al., 2006). van Rijn et al. (2011) recently investigated levels of salivary testosterone and estradiol in male UHR subjects and found lower testosterone levels and normal estradiol levels in male UHR subjects compared with male controls. This suggests potential neuroendocrine markers for UHR patients. The early effect of testosterone on neurodevelopment in patients with schizophrenia was demonstrated in an abnormal ratio between the lengths of the second and fourth digits (2D:4D ratio; Collinson et al., 2010). The ratio is a marker of the amount of prenatal circulating testosterone and represents early organizational effects of testosterone on brain development (Lutchmaya et al., 2004). Taken together, these findings suggest that gonadal hormones protect against the symptoms of schizophrenia. More than a century ago, Kraepelin first proposed that "dementia praecox" may be due to an imbalance of sexual hormones (Kendler and Jablensky, 2011). Based on previous studies, we suggest that vulnerability to psychosis may reflect an imbalance in sexual hormones rather than a deficiency in a single hormone. Specifically, aberrant sex hormones, particularly during adolescence and early adulthood, may affect structural brain abnormalities. 
Structural brain abnormalities in psychosis can be associated with deficits in lipid membrane and essential fatty acids. Previous studies have shown abnormalities in membrane function, including the metabolism and structure of membrane phospholipids involving PUFAs in psychosis. These have been implicated in the membrane hypothesis of schizophrenia (Horrobin et al., 1994; Mossaheb et al., 2012). Specifically, mitochondrial dysfunction due to irregular cellular metabolism and oxidative processes (Clay et al., 2011) and redox/glutathione dysregulation as a result of oxidative stress from dysregulation of glutathione synthesis have been reported (Bokkon and Antal, 2011). The long-chain PUFAs, such as the omega-3 fatty acids, eicosapentaenoic (EPA) and docosahexaenoic (DHA) acids, and the omega- 6 fatty acids such as arachidonic acid (AA), are structural components of neuronal membranes. They play a crucial role in brain function as well as normal growth and development including neuronal growth, dendritic arborization, and synapse formation (Kawakita et al., 2006). Thus, consumption of omega-3 PUFAs may influence brain morphology. Long-chain omega-3 fatty acid intake in healthy adults has been associated positively with corticolimbic GMV in the subgenual ACC, hippocampus, and amygdala (Conklin et al., 2007). However, a recent meta-analysis has clearly indicated PUFA treatment are not effective in schizophrenia (Fusar-Poli and Berger, 2012). Conversely, in UHR patients, a 12 -week intervention with omega- 3 significantly reduced the transition rate to psychosis $(22.6 \%)$ compared with the placebo group. Moreover, the treatment resulted in significant symptomatic and functional improvements during the entire 12-month follow-up period (Amminger et al., 2010). Future studies should investigate the relationship between omega-3 PUFAs and structural GMV with respect to preventing the onset of psychosis in UHR patients.

The contribution of membrane turnover in the prediction of psychosis may also involve using 31P-magnetic resonance spectroscopy (MRS) to measure the phosphomonoesters and phosphodiesters levels that are reflective of membrane phospholipid precursors and breakdown products (Reddy and Keshavan, 2003). The relationship between cerebral morphology and membrane phospholipid metabolism, as measured by phosphorusMRS (31P-MRS), was reported in patients with schizophrenia (Keshavan et al., 1993). Patients with schizophrenia showed altered metabolism of membrane phospholipids during the early course of the illness, which is consistent with a neurodevelopmental abnormality around the critical period of adolescence (Stanley, 2002). Proton-MRS (1H-MRS) has also been used to measure metabolite concentrations, such as choline, creatine, $\mathrm{N}$ acetylaspartate (NAA), glutamine, and glutamate in the brain. In patients at UHR, 1H-MRS studies showed normal levels of NAA, a marker of neuronal/axonal integrity, in the hippocampus of UHR subjects (Wood et al., 2003, 2010). UHR subjects had metabolic changes in the PFC and ACC that were investigated using NAA/creatine and NAA/choline ratios (Wood et al., 2003; Byun et al., 2009). Based on growing evidence for the involvement of glutamatergic abnormalities in schizophrenia, $1 \mathrm{H}$-MRS studies in patients with UHR investigated glutamate levels in the brain. Glutamatergic dysfunction may have an impact on dopaminergic transmission and ultimately lead to the onset of psychosis. Studies have shown an altered relationship between glutamate levels and striatal dopamine function in UHR patients (Stone et al., 2010). Multimodal studies combining MRI and MRS in UHR subjects indicated that glutamatergic dysfunction in the thalamus is associated with a reduction in GMV in the medial temporal cortex and insula (Stone et al., 2009). Thus, imbalanced neurochemicals may underlie structural brain abnormalities in psychosis.

Structural GM abnormalities may be associated with $\mathrm{N}$ methyl-D-aspartate (NMDA) receptor hypofunction, termed the excitotoxic hypothesis of psychosis-induced apoptosis (Farber, 2003). According to this hypothesis, disinhibited excitation of NMDA receptors on gamma-aminobutyric acid (GABA)-ergic interneurons results in excessive stimulation of the class of glutamate receptors and excitotoxicity, including the generation of ROS and apoptotic cell death in hippocampal and cortical areas (Farber, 2003). The involvement of NMDA receptor hypofunction in schizophrenic pathophysiology has been supported by findings that non-competitive NMDA receptor antagonists, phencyclidine (PCP), or ketamine, produce schizophrenia-like symptoms in healthy individuals and profoundly exacerbate pre-existing symptoms in patients with psychosis.

Taken together, these findings suggest that excessive oxidative stress and deficits in the ability to cope with oxidative stress as a result of genetic, epigenetic, and environmental risk factors may result in structural GM abnormalities. Thus, neuroprotective strategies may be used as potential treatments to delay or prevent the progression of the disorder in UHR patients and halt progressive atrophy in patients with psychosis. In this regard, the use of recombinant human erythropoietin (EPO), a neuroprotective hormone (Brines and Cerami, 2005), may improve neuroplasticity (Adamcio et al., 2008) and delay the loss of GMV (Wüstenberg et al., 2011). More recently, low-dose lithium has been shown to have neuroprotective effects on hippocampal microstructure in a small UHR group in a longitudinal MRI-MRS study (Berger et al., 2012).

Considering ethical issues related to drug intervention in subjects at UHR, an interesting option is to target nutrition. Nutrition affects the brain throughout life (Bourre, 2006a,b; Dauncey, 2009). Early nutrition affects brain structures and cognitive function in later life (Isaacs et al., 2008). A recent VBM study investigated whether breakfast foods (i.e., bread vs. rice) affect GMV and cognitive function in healthy children (Taki et al., 2010). The rice group showed higher IQ scores and greater GMV in the STG, inferior frontal gyrus, and caudate nuclei, whereas the bread group displayed greater GMV in the fronto-parietal region. The authors speculated that a possible mechanism might be the difference in the glycemic index (GI) between the two groups; low GI foods are associated with less blood-glucose fluctuation compared with high GI foods.

Long-chain PUFAs and antioxidants protect neurons against oxidative stress. Antioxidants such as vitamins E, C, and A (betacarotene), glutathione, and minerals cannot be synthesized de novo in humans and are therefore required from dietary sources. PUFAs play a crucial role in brain functioning, as well as in normal growth and development. It is important to consider the ratio of omega6/omega-3 fatty acids in the diet (Simopoulos, 2002) as well as the consumption of nutrients that can influence omega-3 status (Berr et al., 2009). Excessive levels of omega- 6 promote the pathogenesis 
of many diseases, including inflammatory and autoimmune diseases, whereas increased levels of omega-3 PUFAs exert suppressive effects (Simopoulos, 2002). Thus, we suggest that nutritional balance and nutrient interactions are important for mental health and brain development. Further studies should focus on nutritional balance in UHR patients and its relationship with GMV.

\section{POTENTIAL ISSUES AND FUTURE DIRECTIONS}

Recent advances in psychiatric research have focused on early detection and prevention of psychosis (McGorry et al., 2009). However, MRI-based biomarkers for UHR patients should be targeted to improve the specificity and sensitivity of criteria that are presently available. Although there have been attempts, to date, the use of MRI-based biomarkers for individuals with psychosis in a clinical setting has not been successful.

First, methodological variability may be a source of heterogeneity across studies (Fusar-Poli et al., 2008a). For example, different scanners (i.e., 1.5 or $3 \mathrm{~T}$ ), different methods of data analysis, and differences in imaging parameters need to be addressed (FusarPoli et al., 2010b). Because a small sample size limits the power to detect differences, there is a growing probability that the results of some studies include false positives. Therefore, multicenter studies are needed to investigate the key comparison between UHR-P and UHR-NP.

Second, antipsychotic exposure is a confounding factor. Previous studies have indicated that antipsychotic treatment in UHR patients may alter GMV in the frontal and temporal areas (Smieskova et al., 2009). Atypical antipsychotic treatment may reverse such alterations (i.e., increased GMV in the inferior frontal gyrus) and prevent the onset of psychosis (Fusar-Poli et al., 2011b). Although recent neuroimaging studies have taken into account the effects of antipsychotics, their effects on the brain cannot be ignored when investigating patients with chronic schizophrenia. Chronic patients generally use antipsychotics. Specifically, the effects of medication exposure should be taken into consideration in studies of patients at UHR-P and UHR-NP.

Third, the criteria used to identify UHR subjects differ across studies. The majority of studies use the Comprehensive Assessment of Symptoms and History (CAARMS; Yung et al., 2005) or the newly developed and shorter Basel Screening Instrument for Psychosis (BSIP; Riecher-Rössler et al., 2007). Other employs the Structured Interview for Prodromal Symptoms (SIPS)/the Scale of Prodromal Symptoms (SOPS) or the basic symptoms (BS) approach. Discrepancies in previous findings may result from differences in trait/state risk factors and BS among UHR individuals for psychosis. However in the recent meta-analysis including over than $2550 \mathrm{HR}$ subjects, the transition risk was comparable across different inclusion criteria (Fusar-Poli et al., 2012b). A further problem is that the UHR group is heterogeneous and typically include both UHR-P and UHR-NP subjects, as well as patients at different levels of risk for psychosis. UHR groups can be divided into two groups based on their symptoms or on the duration of the ARMS status since first presentation. Such divisions might sort patients, respectively, as (i) individuals in the early prodromal state (EPS) with trait vulnerability based on their symptoms and (ii) individuals in the late prodromal state (LPS) with APS and BLIPS (Häfner et al., 1992) or as (i) individuals with short-term ARMS status (ARMS-ST) and (ii) those with long-term ARMS status (ARMS-LT) based on the time since first presentation (McGorry et al., 2009; Smieskova et al., 2012a,b). APS and BLIPS subjects are closer to transition to psychosis than are subjects in the EPS, including trait subjects. Indeed, the intake criteria for UHR subjects predicted transition over 6 months in the order of trait alone $<$ APS $<$ BLIPS (Nelson et al., 2011). Hurlemann et al. (2008) reported that although both EPS and LPS patients had reduced hippocampal GMV, the reductions were correlated with poorer cognitive performance in only the LPS. Koutsouleris et al. (2009a) also investigated differences in GMV between EPS and LPS patients and between UHR-P and UHR-NP groups. They found PFC alterations in UHR-P relative to UHR-NP and controls, which overlapped with the findings in LPS. Jung et al. (2011) found significantly reduced cortical thickness in the STG, PFC, ACC, and parietal area of the group with APS and BLIPS compared with healthy controls. Koutsouleris et al. (2009b) recently distinguished between EPS and LPS using GMV differences between groups with multivariate pattern classification.

A previous study that investigated the differences between ARMS-ST (higher probability) and ARMS-LT (lower probability) improved our understanding of early intervention in the context of a clinical staging model (McGorry et al., 2009). ARMS-ST displayed less GMV in the insula compared with ARMS-LT and FEP. Moreover, these GMV differences were correlated with symptoms (Smieskova et al., 2012b). A multimodal fMRI-VBM approach revealed smaller GMV in the temporal gyrus and extending into the insula in ARMS-ST compared with ARMS-LT. Moreover, a correlation was noted between the reductions in GM and reduced activation in the same area of ARMS-LT (Smieskova et al., 2012c). The authors speculated that the differences between ARMS-ST and ARMS-LT was related to increased vulnerability-associated alterations in ARMS-ST and to resilience or protective processes in ARMS-LT. Further studies should take into account different levels of risk for psychosis to clarify vulnerability-related, disease-related, and resilience biomarkers. It would be interesting to investigate cortical changes associated with the duration of the ARMS status after first presentation in terms of a progressive neurodevelopmental model of schizophrenia. However, that study must note whether patients identified as ARMS-LT were originally non-psychotic (i.e., healthy subjects with false-positive assessment) or remained non-psychotic through their resilience or protective processes.

Fourth, subjects who meet UHR criteria can transition to schizophrenia as well as to other psychiatric disorders including bipolar disorder. This prevents the development of disease-specific prevention strategies and limits the clinical applicability of ongoing basic research (Fusar-Poli et al., 2008b). The criteria are able to identify those at UHR for psychosis but not for schizophrenia. Separate criteria exist for the diagnosis of bipolar disorder (BAR; Bechdolf et al., 2012a). Previous MRI findings in UHR subjects are related to psychosis rather than to schizophrenia. Recent studies have attempted to distinguish UHR subjects who converted to a diagnosis of schizophrenia (UHR-SZ) from other UHR subjects who converted to other disorders. Dazzan et al. (2012) divided UHR subjects into three groups; UHR-SZ, UHR who converted to affective psychosis (UHR-AFF), and UHR-NP. Decreased volume 
in the parietal cortex and a trend toward decreases in the middle temporal and inferior frontal cortices were noted in UHR-SZ subjects, and smaller superior frontal volumes were noted in UHRAFF compared with UHR-NP subjects. Additionally, reduced subcallosal cingulate volume was noted in UHR-AFF subjects compared with UHR-SZ subjects. Bechdolf et al. (2012b) investigated amygdalar, insular, lateral ventricular, and whole-brain volumes in subjects at UHR, all of whom had developed bipolar I or II disorder (UHR-BP) at the time of a follow-up session, compared with UHR-NP and healthy controls. They found reduced amygdalar and insular volumes in UHR-BP subjects compared with UHRNP subjects and controls. An approach that distinguishes UHR-SZ subjects from individuals with other psychosis will enable the development of specific biomarkers to detect and prevent the onset of schizophrenia. Moreover, a thorough understanding of the neurobiological nature of psychiatric diseases, along with knowledge of common and different structural abnormalities before and after the onset of different psychotic disorders, is warranted.

Fifth, it is still unclear to what extent state and genetic factors influence structural brain alterations in patients with psychosis and, particularly, what shared genetic factors in UHR patients influence their structural abnormalities. This information will need to be clarified to improve the feasibility of MRI-based biomarkers. The findings from previous studies that compared EPS with LPS may implicate more state-related abnormalities than do other UHR studies. Few studies have directly compared UHR and GHR. A recent meta-analysis of VBM studies with GHR and antipsychotic-naïve FEP revealed reduced GMV in the parahippocampal gyrus and ACC in GHR compared with controls and decreased volumes in the ACC, precuneus, cerebellum, and STG in FEP compared with GHR (Fusar-Poli et al., 2012e). The authors concluded that reductions in GM in the ACC are markers of genetic liability to psychosis, whereas reductions in the STG and cerebellum are markers of the onset of the illness. Another voxelwise meta-analysis was conducted on VBM studies of subjects at UHR and GHR (Fusar-Poli et al., 2011b). Compared with controls, reductions in GM were observed in the parahippocampal gyrus, STG, insula, and inferior and medial frontal gyri in UHR subjects and in the parahippocampal gyrus and ACC in GHR subjects. Direct comparisons between subjects at UHR and GHR showed that subjects at UHR had less GMV in the ACC and greater GMV in the parahippocampal gyrus, insula, and STG than did GHR subjects.

Sixth, there are only a few longitudinal studies on this topic. Adolescence is a critical period in the development of the human brain, which is particularly vulnerable to the onset of psychosis at this time (Gogtay et al., 2011). Developmental changes in GMV occur at different rates in different brain regions (Sowell et al., 2003; Gogtay et al., 2004). Pantelis et al. (2010) focused on progressive changes in GM in the PFC, insula, and STG throughout the transition to psychosis in longitudinal studies of patients with $\mathrm{UHR}$, as opposed to inconsistent findings from cross-sectional comparisons in the same cohorts. Trajectories of brain development may be more informative than cross-sectional studies of structural abnormalities. These studies suggested acceleration of the normal maturational process in vulnerability to psychosis (Sun et al., 2009a; Gogtay et al., 2011). Many researchers have suggested the heritability of dynamic brain changes in normal individuals and non-affected relatives (Gogtay et al., 2007; Raznahan et al., 2011). If abnormalities in normal development are potential endophenotypes, the concept of the endophenotype in schizophrenia should be redefined (Pantelis et al., 2010). Future studies should clarify the theory that genes associated with brain development during adolescence and early adulthood affect brain abnormalities by accelerating the process of normal neurodevelopment.

Seventh, the effect of sex on brain abnormalities in subjects at UHR remains unclear. The prevalence of psychosis is greater in males throughout most of adulthood, but is equal in males and females by the end of the risk period (MacDonald and Schulz, 2009). Furthermore, sex hormones influence the onset of psychosis and the associated brain abnormalities.

Eighth, a variety of structural MRI studies in subjects at UHR now exist, including single MRI and multi-imaging modalities. Multiple papers have been published on patients at UHR but it is unclear how many subjects overlap in the published studies. Further meta-studies should take into account the direct overlap between subjects.

Finally, the relationship between structural brain abnormalities and cognitive dysfunction during the transition to psychosis is unclear. One recent study investigated the relationship between structure and cognition in UHR patients (Koutsouleris et al., 2010b). Whereas a relationship between executive function and insular GMV was noted in healthy controls, impaired executive function and positive correlations with the cerebellum and negative correlations with the rectus were noted in UHR subjects. A relationship between executive function and caudate volume in UHR subjects has recently been reported (Hannan et al., 2010).

Taken together, these observations indicate that additional longitudinal multimodal studies with larger samples of drug-naïve subjects who convert to different psychoses, conducted across numerous centers and with data from multiple scanners are necessary to address the aforementioned issues. Additionally, the investigation of different features of cortical areas, including structural asymmetries, gyrification, and shape will provide optimal MRI-based biomarkers for psychosis.

\section{CONCLUSION}

Over the past generation, MRI studies in prodromal psychosis have exposed a "smoking gun" with respect to whether there are brain abnormalities and what brain regions are involved in the pathophysiology of psychosis. Indeed, MRI studies represent a marvelous approach that provides new knowledge about the neurobiological trajectories of the disorder. Structural brain abnormalities in psychosis occur prior to full-blown symptoms and progressively worsen over the course of the illness. The discoveries from MRI studies, in combination with evidence from genetic, neurochemical, and environmental studies, are prompting a paradigm shift in psychosis research. These data are contributing to a progressive neurodevelopmental model, i.e., a combined neurodevelopmental and neurodegenerative model (Rapoport and Gogtay, 2011). Furthermore, MRI studies can confirm the safety evaluation and effectiveness of new candidate drugs and non-drug interventions. The identification of regions associated with the 
onset of psychosis may localize targets for treatment interventions. Recent advances in clinical and neuroimaging techniques suggest the feasibility of MRI-based biomarkers for early interventions. Knowledge of metabolic processes that regulate homeostatic balance in the brain can greatly improve the development of new drugs that protect against structural brain abnormalities. All of the hypotheses regarding brain abnormalities associated with the onset of psychosis may converge into the idea that abnormalities in specific brain processes of energy metabolism are affected by genetic and/or environmental factors. In the context of impaired brain energy metabolism, brain abnormalities and psychosis may result from an imbalance of neurochemical factors and nutritional status. In this regard, neuroprotective agents can interrupt

\section{REFERENCES}

Adamcio, B., Sargin, D., Stradomska, A., Medrihan, L., Gertler, C., Theis, F., et al. (2008). Erythropoietin enhances hippocampal long-term potentiation and memory. BMC Biol. 6, 37. doi:10.1186/1741-7007-6-37

Ader, R. (2007). Psychoneuroimmunology, 4th Edn, Vol. 2. New York, NY: Elsevier Academic Press, 563-578.

Akhondzadeh, S., Rezaei, F., Larijani, B., Nejatisafa, A. A., Kashani, L., and Abbasi, S. H. (2006). Correlation between testosterone, gonadotropins and prolactin and severity of negative symptoms in male patients with chronic schizophrenia. Schizophr. Res. 84, 405-410.

Allen, N. D. (2008). Temporal and epigenetic regulation of neurodevelopmental plasticity. Philos. Trans. R. Soc. Lond. B Biol. Sci. 363, 23-38.

Amminger, G. P., Schäfer, M. R., Papageorgiou, K., Klier, C. M., Cotton, S. M., Harrigan, S. M., et al. (2010). Long-chain omega-3 fatty acids for indicated prevention of psychotic disorders: a randomized, placebocontrolled trial. Arch. Gen. Psychiatry 67, 146-154.

Ansell, E. B., Rando, K., Tuit, K., Guarnaccia, J., and Sinha, R. (2012). Cumulative adversity and smaller gray matter volume in medial prefrontal, anterior cingulate, and insula regions. Biol. Psychiatry 72, 57-64.

Bechdolf, A., Ratheesh, A., Wood, S. J., Tecic, T., Conus, P., Nelson, B., et al. (2012a). Rationale and first results of developing atrisk (Prodromal) criteria for bipolar disorder. Curr. Pharm. Des. 18, 358-375.

Bechdolf, A., Wood, S. J., Nelson, B., Velakoulis, D., Yücel, M., Takahashi, T., et al. (2012b). Amygdala and insula volumes prior to illness onset in bipolar disorder: a magnetic resonance imaging study. Psychiatry Res. 201, 34-39.
Behl, C., Widmann, M., Trapp, T., and Holsboer, F. (1995). 17-beta estradiol protects neurons from oxidative stress-induced cell death in vitro. Biochem. Biophys. Res. Commun. 216, 473-482.

Benes, F. M., Majocha, R., Bird, E. D., and Marotta, C. A. (1987). Increased vertical axon numbers in cingulate cortex of schizophrenics. Arch. Gen. Psychiatry 44, 1017-1021.

Berger, G. E., Wood, S. J., Ross, M., Hamer, C. A., Wellard, R. M., Pell, G., et al. (2012). Neuroprotective effects of low-dose lithium in individuals at ultra-high risk for psychosis. a longitudinal MRI/MRS study. Curr. Pharm. Des. 18, 570-575.

Berr, C., Akbaraly, T., Arnaud, J., Hininger, I., Roussel, A. M., and Barberger Gateau, P. (2009). Increased selenium intake in elderly high fish consumers may account for health benefits previously ascribed to omega-3 fatty acids. J. Nutr. Health Aging 13, 14-18.

Blakemore, S. J. (2008). The social brain in adolescence. Nat. Rev. Neurosci. 9, 267-277.

Bleuler, E. (1950). Dementia Praecox or the Group of Schizophrenias, trans. J. Zinkin (New York, NY: International Universities Press).

Bokkon, I., and Antal, I. (2011). Schizophrenia: redox regulation and volume neurotransmission. Curr. Neuropharmacol. 9, 289-300.

Borgwardt, S., McGuire, P., and FusarPoli, P. (2011). Gray matters!mapping the transition to psychosis. Schizophr. Res. 133, 63-67.

Borgwardt, S. J., McGuire, P. K., Aston, J., Gschwandtner, U., Pflüger, M. O., Stieglitz, R. D., et al. (2008). Reductions in frontal, temporal and parietal volume associated with the onset of psychosis. Schizophr. Res. 106, 108-114.

Borgwardt, S. J., Riecher-Rössler, A., Dazzan, P., Chitnis, X., Aston, J., Drewe, M., et al. (2007a). Regional gray matter volume abnormalities

structural brain alterations and symptoms of psychosis. Moreover, nutritional components may affect neuroplasticity. Psychosis vulnerability studies that use neuroimaging techniques will help to bridge the gap between fundamental knowledge of brain abnormalities in psychosis and therapeutic implications for treatment and hence pave the way for new treatment strategies.

\section{ACKNOWLEDGMENTS}

This study was supported by a grant of the Korea Healthcare technology R\&D project, Ministry for Health, Welfare and Family Affairs, Republic of Korea (A090096). The authors thank the faculty and staff of the Clinical Cognitive Neuroscience Center (CCNC) for their help and support.

in the at risk mental state. Biol. Psychiatry 61, 1148-1156.

Borgwardt, S. J., McGuire, P. K., Aston, J., Berger, G., Dazzan, P., Gschwandtner, U., et al. (2007b). Structural brain abnormalities in individuals with an at-risk mental state who later develop psychosis. Br. J. Psychiatry 51, s69-s75.

Bourre, J. M. (2006a). Effects of nutrients (in food) on the structure and function of the nervous system: update on dietary requirements for brain. Part 1: micronutrients. J. Nutr. Health Aging 10, 377-385.

Bourre, J. M. (2006b). Effects of nutrients (in food) on the structure and function of the nervous system: update on dietary requirements for brain. Part 2: macronutrients. J. Nutr. Health Aging 10, 386-399.

Brines, M., and Cerami, A. (2005). Emerging biological roles for erythropoietin in the nervous system. Nat. Rev. Neurosci. 6, 484-494.

Buehlmann, E., Berger, G. E., Aston, J., Gschwandtner, U., Pflueger, M. O., Borgwardt, S. J., et al. (2010). Hippocampus abnormalities in at risk mental states for psychosis? a crosssectional high resolution region of interest magnetic resonance imaging study. J. Psychiatr. Res. 44, 447-453.

Byun, M. S., Choi, J. S., Yoo, S. Y., Kang, D. H., Choi, C. H., Jang, D. P., et al. (2009). Depressive symptoms and brain metabolite alterations in subjects at ultra-high risk for psychosis: a preliminary study. Psychiatry Investig. 6, 264-271.

Chklovskii, D. B., Schikorski, T., and Stevens, C. F. (2002). Wiring optimization in cortical circuits. Neuron 34, 341-347.

Choi, J. S., Park, J. Y., Jung, M. H., Jang, J. H., Kang, D. H., Jung, W. H., et al. (2012). Phase-specific brain change of spatial working memory processing in genetic and ultra-high risk groups of schizophrenia. Schizophr. Bull. 38, 1189-1199.
Chung, Y. S., Kang, D. H., Shin, N. Y., Yoo, S. Y., and Kwon, J. S. (2008). Deficit of theory of mind in individuals at ultra-high-risk for schizophrenia. Schizophr. Res. 99, 111-118.

Clay, H. B., Sillivan, S., and Konradi, C. (2011). Mitochondrial dysfunction and pathology in bipolar disorder and schizophrenia. Int. J. Dev. Neurosci. 29, 311-324.

Collinson, S. L., Lim, M., Chaw, J. H., Verma, S., Sim, K., Rapisarda, A., et al. (2010). Increased ratio of 2 nd to 4th digit (2D:4D) in schizophrenia. Psychiatry Res. 176, 8-12.

Conklin, S. M., Gianaros, P. J., Brown, S. M., Yao, J. K., Hariri, A. R., Manuck, S. B., et al. (2007). Longchain omega-3 fatty acid intake is associated positively with corticolimbic gray matter volume in healthy adults. Neurosci. Lett. 421, 209-212.

Dauncey, M. J. (2009). New insights into nutrition and cognitive neuroscience. Proc. Nutr. Soc. 68, 408-415.

Dazzan, P., Soulsby, B., Mechelli, A., Wood, S. J., Velakoulis, D., Phillips, L. J., et al. (2012). Volumetric abnormalities predating the onset of schizophrenia and affective psychoses: an MRI study in subjects at ultrahigh risk of psychosis. Schizophr. Bull. 38, 1083-1091.

Duggal, H. S., Jagadheesan, K., and Nizamie, S. H. (2002). Acute onset of schizophrenia following autocastration. Can. J. Psychiatry 47, 283-284.

Edmiston, E. E., Wang, F., Mazure, C. M., Guiney, J., Sinha, R., Mayes, L. C., et al. (2011). Corticostriatal-limbic gray matter morphology in adolescents with self-reported exposure to childhood maltreatment. Arch. Pediatr. Adolesc. Med. 165, 1069-1077.

Farber, N. B. (2003). The NMDA receptor hypofunction model of psychosis. Ann. N. Y. Acad. Sci. 1003, 119-130.

Fingerman, K., Berg, C., Smith, J., and Antonucci, T. (2010). Handbook of Life-Span Development. New York, NY: Springer Publishing, 363-383. 
Flores, G., Wood, G. K., Liang, J. J., Quirion, R., and Srivastava, L. K. (1996). Enhanced amphetamine sensitivity and increased expression of dopamine D2 receptors in postpubertal rats after neonatal excitotoxic lesions of the medial prefrontal cortex. J. Neurosci. 16, 7366-7375.

Fornito, A., Yucel, M., Dean, B., Wood, S. J., and Pantelis, C. (2009). Anatomical abnormalities of the anterior cingulate cortex in schizophrenia: bridging the gap between neuroimaging and neuropathology. Schizophr. Bull. 35, 973-993.

Fornito, A., Yung, A. R., Wood, S. J., Phillips, L. J., Nelson, B., Cotton, S., et al. (2008). Anatomic abnormalities of the anterior cingulate cortex before psychosis onset: an MRI study of ultra-high-risk individuals. Biol. Psychiatry 64, 758-765.

Fusar-Poli, P., Allen, P., and McGuire, P. (2008a). Neuroimaging studies of the early stages of psychosis: a critical review. Eur. Psychiatry 23, 237-244.

Fusar-Poli, P., Abbamonte, M., and Borgwardt, S. (2008b). Differential diagnosis between the early onset of schizophrenia and bipolar disorder: potential role of neuroimaging. CNS Spectr. 13, 363-364.

Fusar-Poli, P., and Berger, G. (2012). Eicosapentaneoic acid interventions in schizophrenia: meta-analysis of randomized placebo controlled studies. J. Clin. Psychopharmacol. 32, 179-185.

Fusar-Poli, P., Borgwardt, S., Bechdolf, A., Addington, J., Riecher-Rössler, A., Schultze-Lutter, F., et al. (2012a). The psychosis high risk state: a comprehensive state of the art review. Arch. Gen. Psychiatry in press.

Fusar-Poli, P., Bonoldi, I., Yung, A. R., Borgwardt, S., Kempton, M. J., Valmaggia, L., et al. (2012b). Predicting psychosis: a meta-analysis of transition outcomes in individuals at high clinical risk. Arch. Gen. Psychiatry 69, 1-10.

Fusar-Poli, P., Bechdolf, A., Taylor, M. J., Bonoldi, I., Carpenter, W. T., Yung, A. R., et al. (2012c). At risk for schizophrenic or affective psychosis? A meta-analysis of ICD/DSM diagnostic outcomes in individuals at high clinical risk. Schizophr. Bull. doi:10.1093/schbul/sbs060

Fusar-Poli, P., Deste, G., Smieskova, R., Barlati, S., Yung, A. R., Howes, O., et al. (2012d). Cognitive functioning in prodromal psychosis: a metaanalysis. Arch. Gen. Psychiatry 69, 562-571.

Fusar-Poli, P., Smieskova, R., Serafini, G., Politi, P., and Borgwardt, S. (2012e). Neuroanatomical markers of genetic liability to psychosis and first episode psychosis: a voxelwise meta-analytical comparison. World J. Biol. Psychiatry. doi:10.3019/15622975.2011.630408

Fusar-Poli, P., Radua, J., McGuire, P., and Borgwardt, S. (2012f). Neuroanatomical maps of psychosis onset: voxel-wise meta-analysis of antipsychotic-naive VBM studies. Schizophr. Bull. 38, 1297-1307.

Fusar-Poli, P., and Broome, M. R. (2006). Conceptual issues in psychiatric neuroimaging. Curr. Opin. Psychiatry 19, 608-612.

Fusar-Poli, P., Howes, O. D., Allen, P., Broome, M., Valli, I., Asselin, M. C., et al. (2010a). Abnormal frontostriatal interactions in people with prodromal signs of psychosis: a multimodal imaging study. Arch. Gen. Psychiatry 67, 683-691.

Fusar-Poli, P., Bhattacharyya, S., Allen, P., Crippa, J. A., Borgwardt, S., Martin-Santos, R., et al. (2010b). Effect of image analysis software on neurofunctional activation during processing of emotional human faces. J. Clin. Neurosci. 17, 311-314.

Fusar-Poli, P., McGuire, P., and Borgwardt, S. (2011a). Mapping prodromal psychosis: a critical review of neuroimaging studies. Eur. Psychiatry 27, 181-191.

Fusar-Poli, P., Borgwardt, S., Crescini, A., Deste, G., Kempton, M. J., Lawrie, S., et al. (2011b). Neuroanatomy of vulnerability to psychosis: a voxel-based meta-analysis. Neurosci. Biobehav. Rev. 35, 1175-1185.

Fusar-Poli, P., Broome, M. R., Woolley, J. B., Johns, L. C., Tabraham, P., Bramon, E., et al. (2011c). Altered brain function directly related to structural abnormalities in people at ultra high risk of psychosis: longitudinal VBM-fMRI study. J. Psychiatr. Res. 45, 190-198.

Fusar-Poli, P., Crossley, N., Woolley, J., Carletti, F., Perez-Iglesias, R., Broome, M., et al. (2011d). Gray matter alterations related to P300 abnormalities in subjects at high risk for psychosis: longitudinal MRIEEG study. Neuroimage 55, 320-328.

Fusar-Poli, P., and Meyer-Lindenberg, A. (2012a). Striatal presynaptic dopamine in schizophrenia, part I: meta-analysis of Dopamine Active Transporter (DAT) density. Schizophr. Bull. doi:10.1093/schbul/sbr111 Fusar-Poli, P., and Meyer-Lindenberg, A. (2012b). Striatal presynaptic dopamine in schizophrenia, part II: meta-analysis of $[18 \mathrm{~F}] /[11 \mathrm{C}]$ DOPA PET studies. Schizophr. Bull. doi:10.1093/schbul/sbr180
Fusar-Poli, P., Perez, J., Broome, M., Borgwardt, S., Placentino, A., Caverzasi, E., et al. (2007). Neurofunctional correlates of vulnerability to psychosis: a systematic review and meta-analysis. Neurosci. Biobehav. Rev. 31, 465-484.

Fusar-Poli, P., and Yung, A. (2012). Should attenuated psychosis syndrome be included in the DSM5? The debate. Lancet 379, 591-592.

Garner, B., Pariante, C. M., Wood, S. J., Velakoulis, D., Phillips, L., Soulsby, B., et al. (2005). Pituitary volume predicts future transition to psychosis in individuals at ultra-high risk of developing psychosis. Biol. Psychiatry 58, 417-423.

Gogtay, N., Giedd, J. N., Lusk, L., Hayashi, K. M., Greenstein, D., Vaituzis, A. C., et al. (2004). Dynamic mapping of human cortical development during childhood through early adulthood. Proc. Natl. Acad. Sci. U.S.A. 101, 8174-8179.

Gogtay, N., Greenstein, D., Lenane, M., Clasen, L., Sharp, W., Gochman, P., et al. (2007). Cortical brain development in nonpsychotic siblings of patients with childhood-onset schizophrenia. Arch. Gen. Psychiatry 64, 772-780.

Gogtay, N., Vyas, N. S., Testa, R., Wood, S. J., and Pantelis, C. (2011). Age of onset of schizophrenia: perspectives from structural neuroimaging studies. Schizophr. Bull. 37, 504-513.

Ha, J. W., Jang, J. H., Kang, D. H. Jung, W. H., Kwon, J. S., and Zhang, B. T. (2009). "Gender classification with cortical thickness measurement," in FUZZ-IEEE'09 Proceedings of the 18th International Conference on Fuzzy Systems, Jeju, South Korea, 41-46.

Häfner, H., Riecher-Rössler, A., Hambrecht, M., Maurer, K., Meissner, S., Schmidtke, A., et al. (1992). IRAOS: an instrument for the assessment of onset and early course of schizophrenia. Schizophr. Res. 6, 209-223.

Haller, S., Borgwardt, S. J., Schindler, C., Aston, J., Radue, E. W., and Riecher-Rossler, A. (2009). Can cortical thickness asymmetry analysis contribute to detection of at-risk mental state and first-episode psychosis? A pilot study. Radiology 250 212-221.

Hammond, J., Le, Q., Goodyer, C. Gelfand, M., Trifiro, M., and LeBlanc, A. (2001). Testosteronemediated neuroprotection through the androgen receptor in human primary neurons. J. Neurochem. 77, 1319-1326.

Hannan, K. L., Wood, S. J., Yung, A. R., Velakoulis, D., Phillips, L. J., Soulsby,
B., et al. (2010). Caudate nucleus volume in individuals at ultra-high risk of psychosis: a cross-sectional magnetic resonance imaging study. Psychiatry Res. 182, 223-230.

He, Y., Chen, Z., and Evans, A. (2008). Structural insights into aberrant topological patterns of large-scale cortical networks in Alzheimer's disease. J. Neurosci. 28, 4756-4766.

Horrobin, D. F., Glen, A. I., and Vaddadi, K. (1994). The membrane hypothesis of schizophrenia. Schizophr. Res. 3, 195-207.

Howes, O. D., and Kapur, S. (2009). The dopamine hypothesis of schizophrenia: version III-the final common pathway. Schizophr. Bull. 35, 549-562.

Howes, O. D., Montgomery, A. J., Asselin, M. C., Murray, R. M., Valli, I., Tabraham, P., et al. (2009). Elevated striatal dopamine function linked to prodromal signs of schizophrenia. Arch. Gen. Psychiatry 66, 13-20.

Hurlemann, R., Jessen, F., Wagner, M., Frommann, I., Ruhrmann, S., Brockhaus, A., et al. (2008). Interrelated neuropsychological and anatomical evidence of hippocampal pathology in the at-risk mental state. Psychol. Med. 38, 843-851.

Isaacs, E. B., Gadian, D. G., Sabatini, S. Chong, W. K., Quinn, B. T., Fischl, B. R., et al. (2008). The effect of early human diet on caudate volumes and IQ. Pediatr. Res. 63, 308-314.

Jang, J. H., Shin, N. Y., Shim, G., Park, H. Y., Kim, E., Jang, G. E., et al. (2011). Longitudinal patterns of social functioning and conversion to psychosis in subjects at ultra-high risk. Aust. N. Z. J. Psychiatry 45, 763-770.

Johnstone, E. C., Crow, T. J., Frith, C. D., Husband, J., and Kreel, L. (1976) Cerebral ventricular size and cognitive impairment in chronic schizophrenia. Lancet 2, 924-926.

Jung, W. H., Jang, J. H., Byun, M. S., An, S. K., and Kwon, J. S. (2010). Structural brain alterations in individuals at ultra-high risk for psychosis: a review of magnetic resonance imaging studies and future directions. $J$. Korean Med. Sci. 25, 1700-1709.

Jung, W. H., Kim, J. S., Jang, J. H., Choi, J. S., Jung, M. H., Park, J. Y., et al. (2011). Cortical thickness reduction in individuals at ultra-high-risk for psychosis. Schizophr. Bull. 37, 839-849.

Kapur, S., Mizrahi, R., and Li, M. (2005). From dopamine to salience to psychosis: linking biology, pharmacology and phenomenology of psychosis. Schizophr. Res. 79, 59-68.

Kawakita, E., Hashimoto, M., and Shido, O. (2006). Docosahexaenoic acid 
promotes neurogenesis in vitro and in vivo. Neuroscience 139, 991-997.

Kendler, K. S., and Jablensky, A. (2011). Kraepelin's concept of psychiatric illness. Psychol. Med. 41, 1119-1126.

Keshavan, M. S., Sanders, R. D., Pettegrew, J. W., Dombrowsky, S. M., and Panchalingam, K. S. (1993). Frontal lobe metabolism and cerebral morphology in schizophrenia: 31P MRS and MRI studies. Schizophr. Res. 10, 241-246.

Kim, H. S., Shin, N. Y., Choi, J. S., Jung, M. H., Jang, J. H., Kang, D. H., et al. (2010). Processing of facial configuration in individuals at ultrahigh risk for schizophrenia. Schizophr. Res. 118, 81-87.

Kim, H. S., Shin, N. Y., Jang, J. H., Kim, E., Shim, G., Park, H. Y., et al. (2011). Social cognition and neurocognition as predictors of conversion to psychosis in individuals at ultra-high risk. Schizophr. Res. 130, 170-175.

Kirch, D. G. (1993). Infection and autoimmunity as etiologic factors in schizophrenia: a review and reappraisal. Schizophr. Bull. 19, 355-370.

Kloppel, S., Abdulkadir, A., Jack, C. R. Jr., Koutsouleris, N., MouraoMiranda, J., and Vemuri, P. (2012). Diagnostic neuroimaging across diseases. Neuroimage 61, 457-463.

Koh, Y., Shin, K. S., Kim, J. S., Choi, J. S., Kang, D. H., Jang, J. H., et al. (2011). An MEG study of alpha modulation in patients with schizophrenia and in subjects at high risk of developing psychosis. Schizophr. Res. 126, 36-42.

Koutsouleris, N., Borgwardt, S., Meisenzahl, E. M., Bottlender, R., Moller, H. J., and Riecher-Rossler, A. (2012). Disease prediction in the at-risk mental state for psychosis using neuroanatomical biomarkers: results from the FePsy Study. Schizophr. Bull. 38, 1234-1246.

Koutsouleris, N., Gaser, C., Bottlender, R., Davatzikos, C., Decker, P., Jäger, M., et al. (2010a). Use of neuroanatomical pattern regression to predict the structural brain dynamics of vulnerability and transition to psychosis. Schizophr. Res. 123, 175-187.

Koutsouleris, N., Patschurek-Kliche, K., Scheuerecker, J., Decker, P., Bottlender, R., Schmitt, G., et al. (2010b). Neuroanatomical correlates of executive dysfunction in the at-risk mental state for psychosis. Schizophr. Res. 123, 160-174.

Koutsouleris, N., Schmitt, G. J., Gaser, C., Bottlender, R., Scheuerecker, J., McGuire, P., et al. (2009a). Neuroanatomical correlates of different vulnerability states for psychosis and their clinical outcomes. Br. J. Psychiatry 195, 218-226.

Koutsouleris, N., Meisenzahl, E. M., Davatzikos, C., Bottlender, R., Frodl, T., Scheuerecker, J., et al. (2009b). Use of neuroanatomical pattern classification to identify subjects in at-risk mental states of psychosis and predict disease transition. Arch. Gen. Psychiatry 66, 700-712.

Kraepelin, E. (1919/1971). Dementia Praecox and Paraphrenia, trans. R. M. Barclay (New York, NY: Krieger).

Kulkarni, J., Hayes, E., and Gavrilidis, E. (2012). Hormones and schizophrenia. Curr. Opin. Psychiatry 25, 89-95.

Lodge, D. J., and Grace, A. A. (2007). Aberrant hippocampal activity underlies the dopamine dysregulation in an animal model of schizophrenia. J. Neurosci. 27, 11424-11430.

Lodge, D. J., and Grace, A. A. (2011). Developmental pathology, dopamine, stress and schizophrenia. Int. J. Dev. Neurosci. 29, 207-213.

Lutchmaya, S., Baron-Cohen, S., Raggatt, P., Knickmeyer, R., and Manning, J. T. (2004). 2nd to 4 th digit ratios, fetal testosterone and estradiol. Early Hum. Dev. 77, 23-28.

MacDonald, A. W., and Schulz, S. C. (2009). What we know: findings that every theory of schizophrenia should explain. Schizophr. Bull. 35, 493-508.

McGorry, P. D., Killackey, E., and Yung, A. (2008). Early intervention in psychosis: concepts, evidence and future directions. World Psychiatry 7, 148-156.

McGorry, P. D., Nelson, B., Amminger, G. P., Bechdolf, A., Francey, S. M., Berger, G., et al. (2009). Intervention in individuals at ultra-high risk for psychosis: a review and future directions. J. Clin. Psychiatry 70, 1206-1212.

McGuire, P., Howes, O. D., Stone, J., and Fusar-Poli, P. (2008). Functional neuroimaging in schizophrenia: diagnosis and drug discovery. Trends Pharmacol. Sci. 29, 91-98.

Mechelli, A., Riecher-Rössler, A., Meisenzahl, E. M., Tognin, S., Wood, S. J., Borgwardt, S. J., et al. (2011). Neuroanatomical abnormalities that predate the onset of psychosis: a multicenter study. Arch. Gen. Psychiatry 68, 489-495.

Meisenzahl, E. M., Koutsouleris, N., Gaser, C., Bottlender, R., Schmitt, G. J., McGuire, P., et al. (2008). Structural brain alterations in subjects at high-risk of psychosis: a voxel-based morphometric study. Schizophr. Res. 102, 150-162.
Menon, V. (2011). Large-scale brain networks and psychopathology: a unifying triple network model. Trends Cogn. Sci. (Regul. Ed.) 15, 483-506.

Mossaheb, N., Schloegelhofer, M. Schaefer, M. R., Fusar-Poli, P. Smesny, S., McGorry, P., et al. (2012). Polyunsaturated fatty acids in emerging psychosis. Curr. Pharm. Des. 18, 576-591.

Nelson, B., Fornito, A., Harrison, B. J. Yücel, M., Sass, L. A., Yung, A. R., et al. (2009). A disturbed sense of self in the psychosis prodrome: linking phenomenology and neurobiology. Neurosci. Biobehav. Rev. 33, 807-817.

Nelson, B., Yuen, K., and Yung, A. R. (2011). Ultra high risk (UHR) for psychosis criteria: are there different levels of risk for transition to psychosis? Schizophr. Res. 125, 62-68.

Norbury, R., Cutter, W. J., Compton, J., Robertson, D. M., Craig, M., Whitehead, M., et al. (2003). The neuroprotective effects of estrogen on the aging brain. Exp. Gerontol. 38, 109-117.

Pantelis, C., Velakoulis, D., McGorry, P. D., Wood, S. J., Suckling, J., Phillips, L. J., et al. (2003). Neuroanatomical abnormalities before and after onset of psychosis: a cross-sectional and longitudinal MRI comparison. Lancet 361, 281-288.

Pantelis, C., Wood, S. J., Velakoulis, D., Testa, R., Fontenelle, L. F., and Yucel, M. (2010). Should we redefine the concept of endophenotype in schizophrenia? Rev. Bras. Psiquiatr. 32, 106-107.

Pantelis, C., Yücel, M., Bora, E., Fornito, A., Testa, R., Brewer, W. J., et al. (2009). Neurobiological markers of illness onset in psychosis and schizophrenia: the search for a moving target. Neuropsychol. Rev. 19 385-398.

Paus, T. (2005). Mapping brain maturation and cognitive development during adolescence. Trends Cogn. Sci. (Regul. Ed.) 9, 60-68.

Phillips, L. J., McGorry, P. D., Garner, B., Thompson, K. N., Pantelis, C., Wood, S. J., et al. (2006). Stress, the hippocampus and the hypothalamic-pituitary-adrenal axis: implications for the development of psychotic disorders. Aust. N. Z. J. Psychiatry 40, 725-741.

Phillips, L. J., Velakoulis, D., Pantelis, C., Wood, S., Yuen, H. P., Yung, A. R. et al. (2002). Non-reduction in hippocampal volume is associated with higher risk of psychosis. Schizophr. Res. 58, 145-158.

Rapoport, J. L., and Gogtay, N. (2011). Childhood onset schizophrenia: support for a progressive neurodevelopmental disorder. Int. J. Dev. Neurosci. 29, 251-258.

Raznahan, A., Greenstein, D., Lee, Y., Long, R., Clasen, L., Gochman, P., et al. (2011). Catechol-o-methyl transferase (COMT) val158met polymorphism and adolescent cortical development in patients with childhoodonset schizophrenia, their nonpsychotic siblings, and healthy controls. Neuroimage 57, 1517-1523.

Reddy, R., and Keshavan, M. S. (2003). Phosphorus magnetic resonance spectroscopy: its utility in examining the membrane hypothesis of schizophrenia. Prostaglandins Leukot. Essent. Fatty Acids 69, 401-405.

Riecher-Rössler, A., Gschwandtner, U., Aston, J., Borgwardt, S., Drewe, M., Fuhr, P., et al. (2007). The Basel early-detection-of-psychosis (FEPSY)-study-design and preliminary results. Acta Psychiatr. Scand. 115, 114-125.

Riecher-Rossler, A., and Kulkarni, J. (2011). Estrogens and gonadal function in schizophrenia and related psychoses. Curr. Top. Behav. Neurosci. 8, 155-171.

Shenton, M. E., Dickey, C. C., Frumin, M., and McCarley, R. W. (2001). A review of MRI findings in schizophrenia. Schizophr. Res. 49, 1-52.

Shim, G., Kang, D. H., Choi, J. S., Jung, M. H., Kwon, S. J., Jang, G. E., et al. (2008). Prospective outcome of early intervention for individuals at ultrahigh-risk for psychosis. Early Interv. Psychiatry 2, 277-284.

Shim, G., Oh, J. S., Jung, W. H., Jang, J. H., Choi, C. H., Kim, E., et al. (2010). Altered resting-state connectivity in subjects at ultra-high risk for psychosis: an fMRI study. Behav. Brain Funct. 6, 58.

Shin, K. S., Kim, J. S., Kang, D. H., Koh, Y., Choi, J. S., O’Donnell, B. F., et al. (2009). Pre-attentive auditory processing in ultra-high-risk for schizophrenia with magnetoencephalography. Biol. Psychiatry 65, 1071-1078.

Shin, K. S., Kim, J. S., Kim, S. N., Koh, Y., Jang, J. H., An, S. K., et al. (2012). Aberrant auditory processing in schizophrenia and in subjects at ultra-high-risk for psychosis. Schizophr. Bull. 38, 1258-1267.

Shin, Y. W., Na, M. H., Ha, T. H., Kang, D. H., Yoo, S. Y., and Kwon, J. S. (2008). Dysfunction in configural face processing in patients with schizophrenia. Schizophr. Bull. 34, 538-543.

Simopoulos, A. P. (2002). The importance of the ratio of omega-6/omega-3 essential fatty 
acids. Biomed. Pharmacother. 56, 365-379.

Simpkins, J. W., Singh, M., and Bishop, J. (1994). The potential role for estrogen replacement therapy in the treatment of the cognitive decline and neurodegeneration associated with Alzheimer's disease. Neurobiol. Aging 15(Suppl. 2), S195-S197.

Smieskova, R., Fusar-Poli, P., Allen, P., Bendfeldt, K., Stieglitz, R. D., Drewe, J., et al. (2009). The effects of antipsychotics on the brain: what have we learnt from structural imaging of schizophrenia? A systematic review. Curr. Pharm. Des. 15, 2535-2549.

Smieskova, R., Fusar-Poli, P., Allen, P., Bendfeldt, K., Stieglitz, R. D., Drewe, J., et al. (2010). Neuroimaging predictors of transition to psychosis - a systematic review and meta-analysis. Neurosci. Biobehav. Rev. 34, 1207-1222.

Smieskova, R., Fusar-Poli, P., RiecherRossler, A., and Borgwardt, S. (2012a). Neuroimaging and resilience factors - staging of the at-risk mental state? Curr. Pharm. Des. 18, 416-421.

Smieskova, R., Fusar-Poli, P., Aston, J., Simon, A., Bendfeldt, K., Lenz, C., et al. (2012b). Insular volume abnormalities associated with different transition probabilities to psychosis. Psychol. Med. 42, 1613-1625.

Smieskova, R., Allen, P., Simon, A., Aston, J., Bendfeldt, K., Drewe, J., et al. (2012c). Different duration of at-risk mental state associated with neurofunctional abnormalities. a multimodal imaging study. Hum. Brain Mapp. 33, 2281-2294.

Smith, R. C., Calderon, M., Ravichandran, G. K., Largen, J., Vroulis, G., Shvartsburd, A., et al. (1984). Nuclear magnetic resonance in schizophrenia: a preliminary study. Psychiatry Res. 12, 137-147.

Sowell, E. R., Peterson, B. S., Thompson, P. M., Welcome, S. E., Henkenius, A. L., and Toga, A. W. (2003). Mapping cortical change across the human life span. Nat. Neurosci. 6, 309-315.

Stanley, J. A. (2002). In vivo magnetic resonance spectroscopy and its application to neuropsychiatric disorders. Can. J. Psychiatry 47, 315-326.

Steen, R. G., Mull, C., McClure, R., Hamer, R. M., and Lieberman, J. A. (2006). Brain volume in firstepisode schizophrenia: systematic review and meta-analysis of magnetic resonance imaging studies. $\mathrm{Br}$. J. Psychiatry 188, 510-518.

Stone, J. M., Day, F., Tsagaraki, H., Valli, I., McLean, M. A., Lythgoe, D. J., et al. (2009). Glutamate dysfunction in people with prodromal symptoms of psychosis: relationship to gray matter volume. Biol. Psychiatry 66, 533-539.

Stone, J. M., Howes, O. D., Egerton, A., Kambeitz, J., Allen, P., Lythgoe, D. J., et al. (2010). Altered relationship between hippocampal glutamate levels and striatal dopamine function in subjects at ultra high risk of psychosis. Biol. Psychiatry 68, 599-602.

Sun, D., Phillips, L., Velakoulis, D., Yung, A., McGorry, P. D., Wood, S. J., et al. (2009a). Progressive brain structural changes mapped as psychosis develops in 'at risk' individuals. Schizophr. Res. 108, 85-92.

Sun, J., Maller, J. J., Guo, L., and Fitzgerald, P. B. (2009b). Superior temporal gyrus volume change in schizophrenia: a review on region of interest volumetric studies. Brain Res. Rev. $61,14-32$.

Takahashi, T., Wood, S. J., Yung, A. R., Soulsby, B., McGorry, P. D., Suzuki, M., et al. (2009a). Progressive gray matter reduction of the superior temporal gyrus during transition to psychosis. Arch. Gen. Psychiatry 66, 366-376.

Takahashi, T., Wood, S. J., Yung, A. R., Phillips, L. J., Soulsby, B., McGorry, P. D., et al. (2009b). Insular cortex gray matter changes in individuals at ultra-high-risk of developing psychosis. Schizophr. Res. 111, 94-102.

Takahashi, T., Wood, S. J., Yung, A. R., Walterfang, M., Phillips, L. J., Soulsby, B., et al. (2010). Superior temporal gyrus volume in antipsychotic-naive people at risk of psychosis. Br. J. Psychiatry 196, 206-211.

Taki, Y., Hashizume, H., Sassa, Y., Takeuchi, H., Asano, M., Asano, K., et al. (2010). Breakfast staple types affect brain gray matter volume and cognitive function in healthy children. PLoS ONE 5, e15213. doi:10.1371/journal.pone.0015213

Thompson, K. N., Phillips, L. J., Komesaroff, P., Yuen, H. P., Wood, S. J., Pantelis, C., et al. (2007). Stress and HPA-axis functioning in young people at ultra high risk for psychosis. J. Psychiatr. Res. 41, 561-569.

van Rijn, S., Aleman, A., de Sonneville, L., Sprong, M., Ziermans, T., Schothorst, P., et al. (2011). Neuroendocrine markers of high risk for psychosis: salivary testosterone in adolescent boys with prodromal symptoms. Psychol. Med. 41, 1815-1822.

Velakoulis, D., Wood, S. J., Wong, M. T., McGorry, P. D., Yung, A., Phillips, L., et al. (2006). Hippocampal and amygdala volumes according to psychosis stage and diagnosis: a magnetic resonance imaging study of chronic schizophrenia, first-episode psychosis, and ultra-high-risk individuals. Arch. Gen. Psychiatry 63, 139-149.

Vigil, P., Orellana, R. F., Cortes, M. E., Molina, C. T., Switzer, B. E., and Klaus, H. (2011). Endocrine modulation of the adolescent brain: a review. J. Pediatr. Adolesc. Gynecol. 24, 330-337.

Whitford, T. J., Wood, S. J., Yung, A., Cocchi, L., Berger, G., Shenton, M. E., et al. (2012). Structural abnormalities in the cuneus associated with herpes simplex virus (type 1) infection in people at ultra high risk of developing psychosis. Schizophr. Res. 135, 175-180.

Witthaus, H., Kaufmann, C., Bohner, G. Ozgürdal, S., Gudlowski, Y., Gallinat, J., et al. (2009). Gray matter abnormalities in subjects at ultra-high risk for schizophrenia and first-episode schizophrenic patients compared to healthy controls. Psychiatry Res. 173, 163-169.

Witthaus, H., Mendes, U., Brüne, M., Ozgürdal, S., Bohner, G., Gudlowski, Y., et al. (2010). Hippocampal subdivision and amygdalar volumes in patients in an at-risk mental state for schizophrenia. J. Psychiatry Neurosci. 35, 33-40.

Wood, S. J., Berger, G., Velakoulis, D., Phillips, L. J., McGorry, P. D., Yung, A. R., et al. (2003). Proton magnetic resonance spectroscopy in first episode psychosis and ultra highrisk individuals. Schizophr. Bull. 29, 831-843.

Wood, S. J., Kennedy, D., Phillips, L. J., Seal, M. L., Yücel, M., Nelson, B., et al. (2010). Hippocampal pathology in individuals at ultra-high risk for psychosis: a multi-modal magnetic resonance study. Neuroimage 52, 62-68.

Wood, S. J., Pantelis, C., Velakoulis, D. Yucel, M., Fornito, A., and McGorry, P. D. (2008). Progressive changes in the development toward schizophrenia: studies in subjects at increased symptomatic risk. Schizophr. Bull. 34, 322-329.

Wood, S. J., Yücel, M., Velakoulis, D., Phillips, L. J., Yung, A. R., Brewer, W., et al. (2005). Hippocampal and anterior cingulate morphology in subjects at ultra-high-risk for psychosis: the role of family history of psychotic illness. Schizophr. Res. 75, 295-301.

Wüstenberg, T., Begemann, M., Bartels, C., Gefeller, O., Stawicki, S., HinzeSelch, D., et al. (2011). Recombinant human erythropoietin delays loss of gray matter in chronic schizophrenia. Mol. Psychiatry 16, 26-36.

Yücel, M., Wood, S. J., Phillips, L. J., Stuart, G. W., Smith, D. J., Yung, A., et al. (2003). Morphology of the anterior cingulate cortex in young men at ultra-high risk of developing a psychotic illness. Br. J. Psychiatry 182, 518-524.

Yung, A. R., and McGorry, P. D. (1996). The prodromal phase of first-episode psychosis: past and current conceptualizations. Schizophr. Bull. 22, 353-370.

Yung, A. R., Phillips, L. J., Yuen, H. P., Francey, S. M., McFarlane, C. A., Hallgren, M., et al. (2003). Psychosis prediction: 12-month follow up of a high-risk ("prodromal") group. Schizophr. Res. 60, 21-32.

Yung, A. R., Yuen, H. P., McGorry, P. D., Phillips, L. J., Kelly, D., Dell'Olio, M., et al. (2005). Mapping the onset of psychosis: the comprehensive assessment of at-risk mental states. Aust. N. Z. J. Psychiatry 39, 964-971.

Ziermans, T. B., Durston, S., Sprong, M., Nederveen, H., van Haren, N. E., Schnack, H. G., et al. (2009). No evidence for structural brain changes in young adolescents at ultra high risk for psychosis. Schizophr. Res. 112, $1-6$.

Ziermans, T. B., Schothorst, P. F., Schnack, H. G., Koolschijn, P. C., Kahn, R. S., van Engeland, H., et al. (2012). Progressive structural brain changes during development of psychosis. Schizophr. Bull. 38, 519-530.

Conflict of Interest Statement: The authors declare that the research was conducted in the absence of any commercial or financial relationships that could be construed as a potential conflict of interest.

Received: 30 July 2012; paper pending published: 03 November 2012; accepted: 06 November 2012; published online: 03 December 2012.

Citation: Jung WH, Borgwardt S, FusarPoli $P$ and Kwon JS (2012) Gray matter volumetric abnormalities associated with the onset of psychosis. Front. Psychiatry 3:101. doi: 10.3389/fpsyt.2012.00101

This article was submitted to Frontiers in Schizophrenia, a specialty of Frontiers in Psychiatry.

Copyright (c) 2012 Jung, Borgwardt, Fusar-Poli and Kwon. This is an openaccess article distributed under the terms of the Creative Commons Attribution License, which permits use, distribution and reproduction in other forums, provided the original authors and source are credited and subject to any copyright notices concerning any third-party graphics etc. 